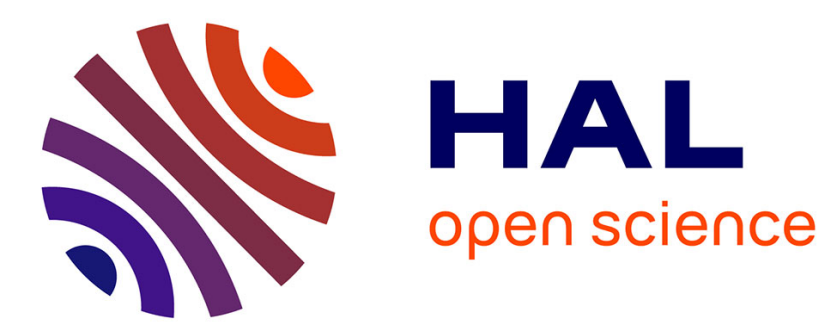

\title{
Critical crossover functions for simple fluids: non-analytical scaling determination of the ising-like crossover paramater
}

Yves Garrabos, Carole Lecoutre, Samuel Marre, Bernard Le Neindre

\section{- To cite this version:}

Yves Garrabos, Carole Lecoutre, Samuel Marre, Bernard Le Neindre. Critical crossover functions for simple fluids: non-analytical scaling determination of the ising-like crossover paramater. Journal of Statistical Physics, 2016, 164 (3), pp.575-615. 10.1007/s10955-016-1554-4 . hal-01344693

\section{HAL Id: hal-01344693 \\ https://hal.science/hal-01344693}

Submitted on 18 Jan 2021

HAL is a multi-disciplinary open access archive for the deposit and dissemination of scientific research documents, whether they are published or not. The documents may come from teaching and research institutions in France or abroad, or from public or private research centers.
L'archive ouverte pluridisciplinaire HAL, est destinée au dépôt et à la diffusion de documents scientifiques de niveau recherche, publiés ou non, émanant des établissements d'enseignement et de recherche français ou étrangers, des laboratoires publics ou privés. 


\title{
Critical Crossover Functions for Simple Fluids: Non-Analytical Scaling Determination of the Ising-Like Crossover Parameter
}

\author{
Yves Garrabos $^{1,2}$ - Carole Lecoutre ${ }^{1,2}$. \\ Samuel Marre ${ }^{1,2}$. Bernard LeNeindre ${ }^{3,4}$
}

\begin{abstract}
A non-analytical scaling determination of the Ising-like crossover parameter is proposed considering the critical isochore of a simple fluid at finite distance from its critical temperature. The mean crossover functions, estimated from the bounded results of the massive renormalization scheme in field theory applied to the $\left(\Phi^{2}\right)_{d}^{2}(n)$ model in three dimensions $(d=3)$ and scalar order parameter $(n=1)$, are used to formulate the corresponding scaling equations valid in two well-defined temperature ranges from the critical temperature. The validity range and the Ising-like nature of the corresponding crossover description are discussed in terms of a single Ising-like scale factor characterizing the critical isochore. The asymptotic value of this scale factor can be predicted within the Ising-like preasymptotic domain. Unfortunately, the absence of precise experimental data in such a close vicinity of the critical point leads the direct testing impossible. A contrario, from our scaling equations and the use of precise measurements performed at finite distance from the critical point, its local value can be estimated beyond the Ising-like preasymptotic domain. This non-analytical scaling determination only needs to make reference to the universal features estimated from the mean crossover functions and to introduce a single master dimensionless length common to all the simple fluids. This latter parameter guaranties the uniqueness of the physical length unit used for the theoretical crossover functions and the fluid singular properties when the generalized critical coordinates of the vapor-liquid critical point of each fluid are known. Xenon case along its critical isochore is considered as a typical example to demonstrate the
\end{abstract}

\footnotetext{
$凶$ Yves Garrabos

yves.garrabos@icmcb.cnrs.fr

Bernard LeNeindre

bernard.leneindre@1spm.cnrs.fr

1 CNRS, Institut de Chimie de la Matière Condensée de Bordeaux - UPR 9048, 33608 Pessac Cedex, France

2 Université de Bordeaux, ICMCB-UPR 9048, 33608 Pessac Cedex, France

3 CNRS, Laboratoire des Sciences des Procédés et des Matériaux- UPR 3407, 93430 Villetaneuse, France

4 Université Paris 13 - Sorbonne Paris Cité, 93430 Villetaneuse, France
} 
singleness of the Ising-like crossover parameter. With the measurements at finite temperature range of the effective singular behaviors of the isothermal compressibility in the homogeneous domain, and the vapor-liquid coexisting densities in the non homogeneous domain, our scaling equations provide the local estimate of this crossover parameter as a function of the temperature distance from the critical temperature. Our results compare favorably with the single asymptotic value calculated only using four critical coordinates of xenon.

Keywords Critical crossover function $\cdot$ Crossover parameter $\cdot$ Critical isochore $\cdot$ Xenon

\section{Introduction}

It is now well-established that the universal features of one-component fluids close to their vapor-liquid critical point are Ising-like in nature [1], i.e., conform with the singular universality close to the non-trivial fixed point of the so-called $\left(\phi^{2}\right)_{d=3}^{2}(n=1)$ model [2-5] ( $d$ and $n$ are respectively the dimensions of the space and the order parameter density). These Isinglike universal features are only related to the contributions of two relevant scaling fields and a single irrelevant scaling field [6,7]. The leading singular behaviors [5] are then governed by the universal values [8] of two independent critical exponents, while the contribution of the confluent corrections to scaling is governed by the universal lowest value [8] of the confluent exponent, noted $\Delta$ below. This simplest theoretical asymptotic description, strictly valid within the so-called Ising-like preasymptotic domain (PAD), is predicted by the renormalization group theory from the estimation of the classical-to-critical crossover functions [9] along the renormalized trajectory which joints the trivial (Gaussian) fixed point to the non-trivial (Wilson-Fisher) fixed point (for details see [5]). As a main result of present concern, the asymptotic singular behavior of the fluid property $P_{s \text {, expt }}^{*}$ along the critical isochore can be approximated by its following restricted two-term form of each infinite Wegner-like expansion [10]

$$
P_{s, \text { expt }}^{*}\left(\left|\Delta \tau^{*}\right|\right)=\Pi_{P}^{ \pm}\left(\left|\Delta \tau^{*}\right|\right)^{-\pi_{P}}\left[1+a_{P}^{ \pm}\left(\left|\Delta \tau^{*}\right|\right)^{\Delta}\right]
$$

where $\pi_{P}$ is the universal critical exponent which governs the Ising-like asymptotic power law behavior of $P_{s, \text { expt }}^{*}$. The amplitudes $\Pi_{P}^{ \pm}$of the leading terms and $a_{P}^{ \pm}$of the first-order terms of the confluent corrections depend on the selected fluid (noted $f$ below). $\Delta \tau^{*}=\frac{T}{T_{c}}-1$ is the reduced temperature distance from the critical temperature $T_{c}$. In the case of fluid properties interested in, we note that a generic additive background term is either subleading with respect to the terms in Eq. (1), or is absent. In Eq. (1), the superscript $*$ labels a nondimensional quantity. The indices + and - indicate respectively the single, high- and the two, low-temperature phase regions. $\Delta=\omega \nu$ is the universal confluent exponent. $\omega$ is the lowest universal correction exponent related to the scaling contribution of an irrelevant field. $v$ is the universal critical exponent which governs the Ising-like asymptotic power law behavior of the correlation length $\xi_{\text {expt }}^{*}$.

The PAD extension where Eq. (1) is valid corresponds to $\left|\Delta \tau^{*}\right| \leq \mathcal{L}_{\mathrm{PAD}}^{f}$ [5]. As currently mentioned in the literature since the 70 's, $\mathcal{L}_{\mathrm{PAD}}^{f}$ is a fluid-dependent parameter claimed small $\left(\mathcal{L}_{\mathrm{PAD}}^{f} \ll 1\right)$ but generally of unknown value, except from the use of the phenomenological master forms [11] of the mean crossover functions estimated from the massive renormalization (MR) scheme. 
Due to the universal scaling laws, only two critical exponents are independent (among the complete $\left\{\pi_{P}\right\}$ set), while the lowest value of the correction exponent $\omega$ accounts for all the confluent crossover corrections, leading to the three-exponent characterization of the theoretical $O(1)$ universality class. Correlatively, due to the universal combinations and ratios between the $\Pi_{P}^{ \pm}$amplitudes (the so-called two-scale-factor universality) on the one hand and the universal ratios between the $a_{P}^{ \pm}$amplitudes on the other, only two leading asymptotic amplitudes among the complete $\left\{\Pi_{P}^{ \pm}\right\}$set and a single confluent amplitude among the complete $\left\{a_{P}^{ \pm}\right\}$set make up the three fluid-dependent parameters which characterize the Ising-like fluid behavior within the PAD. Therefore, the knowledge of $\mathcal{L}_{\text {PAD }}^{f}$ appears as the essential non-universal parameter to validate the three (two leading and one confluent) amplitudes characterization along this primary critical path of each Ising-like critical fluid $[7,9,11]$.

Assuming the existence of the above Ising-like three-amplitude characterization, the problem of using renormalization group theory to develop the theoretical descriptions of the crossover from the Ising-like critical behavior asymptotically close to the critical point to the mean-field thermodynamic behavior away from the critical point has in principle been solved about three decades ago [3,12-15]. However, for the crossover functions calculated from the massive renormalization (MR) scheme [3], or the minimal-substraction renormalization (MSR) scheme [12,13], as well as for the parametric forms of the scaled equations of state $[14,15]$, the fits [16-19] used the experimental data obtained at finite temperature distance from $T_{c}$, i.e., in a temperature range well-beyond the PAD extension where Eq. (1) remains valid. In such a case, the analytical backgrounds as well as the classical-to-critical crossover behavior due to the Gaussian critical point further hindered the test of the asymptotic Isinglike fluid behavior of Eq. (1) when approaching the non-trivial critical point. The observed crossover behavior in one-component fluids needs to account for higher-order terms of the Wegner-like expansions in order to carefully fit the measurements performed far away from critical temperature. The unavoidable implicit constraint $1 \gg \Delta \tau^{*}>\mathcal{L}_{\mathrm{PAD}}^{f}$ in the data fitting analyses performed at finite distance from the vapor-liquid critical point, leads to the practical definition of the so-called extended asymptotic domain (EAD) $\mathcal{L}_{\mathrm{PAD}}^{f}<\Delta \tau^{*} \leq \mathcal{L}_{\mathrm{EAD}}^{f}$ where the fit is thus claimed to be correct. In such a non-asymptotic domain, the choice of the functional forms for fitting the data obtained within the EAD remains a puzzle for the experimentalists whose objective is to find the fluid-dependent amplitudes characterizing the PAD description (see Refs. [20,21] for an illustrative example). More generally, on the basis of Wegner-like expansions alone, it still seems today difficult to manage the exponent values of the confluent corrections due to several irrelevant or nonconfluent fields, while it remains not easy to a priori select a restricted number of significants terms in the infinite, low-convergent Wegner-like expansions [10]. It was then necessary to fix the universal values of the critical exponents and amplitude combinations to their Ising-like theoretical estimates to observe that the existing theories provide a good description of the experimental thermodynamic property data of many fluids, not only along the critical isochore [16-19], but also in the entire thermodynamic plane as reviewed by Behnejad et al. [22] from the various use of the parametric equations of state.

Therefore, the resulting fitting values of the amplitudes involved in the two-term asymptotic Eq. (1) were always interpolated values, i.e., highly dependent of the exact estimation of the Ising-like critical limit and the number of the free parameters which are introduced in the crossover theory to fit the experimental data at finite distance from the critical point. For instance, the universal asymptotic description of the PAD from the upgraded MR crossover functions needs to briefly be replaced in the context of the recent theoretical achievements 
related to the corresponding Ising-like universal estimates. An extensive set of universal values of the exponents and the amplitude combinations has been published, as shown for example in Refs. [23-25]. These most recent results are useful for the comparison between different theoretical asymptotic methods. A reader interested in such a detailed comparison between the results obtained using the different theoretical methods can use Tables I and II of Ref. [23] and Tables 3 and 4 of Ref. [24], or the summarizing Tables 5 and 12 of Ref. [25], and can refer to the extensive list of references quoted by the authors.

However, the actual estimates of universal features extracted from the existing experimental data cannot reach such theoretical levels of accuracy. Moreover, integrating (and then fixing) the most precise theoretical estimates of Ising-like universal exponents, amplitude ratios, and universal features of the sub-leading confluent correction terms in resummed critical-to-classical crossover functions, or in parametric forms of the equations of state, remains a theoretical challenge. It is plausible that the amplitudes of the corrections to scaling in the theoretical methods have a residual impact on the accurate estimates of universal quantities based on quite different models, in particular in the case of the correction exponent $\omega$. As a consequence, any resummation process of the infinite Wegner-like expansions needs to precisely account for the error-bar propagations approaching the non-trivial critical temperature when the leading and subleading terms are included. Accordingly, from the MR renormalization scheme along the primary path with $h=0$ that incorporates the contribution of a single irrelevant field characterized by $\Delta$, the interpolated results for the PAD description are provided by the min and max estimates [5] of the upgraded crossover functions, despite the probable underestimations of the errors using field-theory critical exponents values. De facto, only such (resummed or implicit) calculated theoretical functional forms of the infinite Wegner-like expansions, estimated along the renormalized trajectory, can be used to fit the fluid data at finite distance to $T_{c}$ introducing a controlled limited number (three) of free nonuniversal parameters. From a crossover theory based on the parametric equation of state, the practical results of the interpolated PAD description with three-amplitude characterization of $f$ are not so easy to analyze due to the effective number of incorporated free parameters larger than three. This effective number is needed to account for instance for the strength and shape of the physical crossover [14], as well as the small contribution of the non-symmetrical order parameter [1] and the hypothetized asymmetric fluid criticality accounted for through the mixing of the physical fields [26]. Nevertheless, since about 25 years, the fitting values of the leading amplitudes, such as $\Pi_{P}^{ \pm}$in Eq. (1), seem to be conform with the two-scale-factor universality of the Ising-like systems. Accordingly, the positive, non-zero fitting values of the amplitudes of the leading corrections to scaling appear as a well-established experimental feature in simple fluids, such as $a_{P}^{ \pm}>0$ in Eq. (1), despite the large uncertainties ( $\left.\sim 50 \%\right)$ in the corresponding interpolated values of $a_{P}^{ \pm} \sim 1$ or some inconsistency [27] in the resulting estimation of the confluent universal ratios.

It is then essential to note that an alternative approach of the Ising-like PAD description conform with the above three-amplitude characterization can be made without any free fluiddependent parameters for a one-component fluid whose the generalized critical coordinates of the gas-liquid critical point are known [11]. Indeed, in such a primary path where the Ising-like theoretical limit is well-defined, the ad hoc mean estimates of these crossover functions given in Ref. [9] were consistent with all the universal features expected from the field theoretical method, especially the universal values of the leading confluent amplitude ratios. Moreover, by construction of the three-term multiplicative form, the mean crossover functions given in Ref. [9] provide a single Ising-like PAD description self-consistent with the calculated universal quantities of Ref. [8]. The singular behavior of each one-component fluid approaching the vapor-liquid critical point must then be predicted only using two fluid- 
dependent scale factors (noted $\vartheta, \psi_{\rho}$ ) for the relevant fields and a single critical wavelength (noted $g_{0}$ ), which can be well-defined in terms of the four generalized critical coordinates (noted $T_{c}, p_{c}, \rho_{c}$ and $\gamma_{c}^{\prime}$ ) of the gas-liquid critical point, as recently analyzed in the xenon isothermal susceptibility case [21]. The determination of $\vartheta, \psi_{\rho}$, and $g_{0}$ is made without any adjustable parameter and takes advantage of the Ising-like master singular behavior of the one-component fluid subclass described by the master crossover functions defined in Ref. [11]. As a result, the complete sets of leading amplitudes [such as $\Pi_{P}^{ \pm}$in Eq. (1)] and confluent amplitudes [such as $a_{P}^{ \pm}$in Eq. (1)], are calculated using $\vartheta, \psi_{\rho}$, and $g_{0}$. The Isinglike PAD description characterized by Eq. (1) is thus accomplished through the noticeable exchange between the three selected amplitudes of Eq. (1) and the three nonuniversal parameters introduced in the crossover functions. Moreover, the knowledge of $\vartheta$ also infers the unequivocal estimation of $\mathcal{L}_{\mathrm{PAD}}^{f}$, which means that the two-term singular behaviors, as well as their temperature extension are well known. Finally, the Ising-like PAD description only requires knowledge of the location of the vapor-liquid critical point in the experimental phase surface of $f$, as initially postulated in Refs. [28-30].

Therefore, the main objective of the present work is not to develop a new crossover theory, but to show that the three parameter characterization of the fluid PAD description without adjustable parameter can numerically be validated by using the mean crossover functions at finite distance from $T_{c}$ along the critical isochore. The improvement over the currently available understanding in the fluid crossover problem in a nonasymptotic situation is thus the derivation of the nonanalytical scaling equations incorporating a single local crossover parameter (noted $\vartheta_{\mathcal{L}}$ below). The resulting local value of this crossover parameter can be used to control the Ising-like extent of the known three-parameter PAD description, asymptotically valid along the primary path. As this control is made numerically without adjustable parameter, it is thus essential to quote the theoretical values of the exponents and amplitudes with quite a large number of digits in order to maintain the required resolution from the error-bar estimates of the min and max results given in Ref. [5]. In addition, performing this control along the primary critical path where the Ising-like critical limit is well-defined is of great importance to probe the singleness of the Ising-like crossover parameter which was never previously demonstrated without the use of free fluid-dependent parameters.

Correlatively, incorporating more than three free parameters in a crossover theory differs from the above predictive approach without adjustable parameter applied to the onecomponent fluid subclass with similar short-ranged molecular interaction. More than three nonuniversal parameters generally leads to unavoidable implicit intercorrelations in the resulting characterization for the expected PAD behavior of the physical systems belonging to the $O$ (1) universality class. In the case of simple fluids, such intercorrelations cannot be controlled due to the absence of precise experimental data in the PAD range closest to the critical point (in addition to the uncontrolled supplementary effect accounting for the hypothetized asymmetric fluid criticality). A fourth non-universal crossover parameter can for instance be related to the contributions of one supplementary irrelevant field. In such a case, the addition of another confluent exponent in the Wegner expansions seems needed to maintain the theoretical coherence. Its can also be related to a more complex physical understanding of the microscopic nature introduced in the model, such as for example, an additional reference to a mesoscopic length or a molecular modification of the range of interaction, as in the numerical description [31] of the results for the three-dimensional Ising model. Such a microscopic complexity does not exist in simple fluids where the (Lennard-Jones-like) molecular interactions are certainly short-ranged. Assuming the existence of only two independent relevant fields and a single irrelevant field in the case of such simple fluids, the critical crossover limit 
is thus expected to be universal, except only three nonuniversal parameters characterizing each simple fluid. At the opposite, going away from the vapor-liquid critical point to reach a hypothetized classical critical point is out of the scope of our modelling strategy to understand the Ising-like nature of the simple fluids without adjustable parameter. Indeed, since the 80's until today, no theoretical progress has been able to correctly account for the abrupt physical crossover behavior observed beyond the extended critical asymptotic domain when $\xi_{\text {expt }}\left(\left|\Delta \tau^{*}\right|\right) \simeq \alpha_{c}$. It is illustrated by the narrow temperature range where the true crossover behavior occurs for the effective exponents $\gamma_{e}$ (see Fig. 1 of Ref. [16] for the isothermal compressibility case) and $\beta_{e}$ (see Fig. 3 of Ref. [32] for the order parameter density case).

In the present work, the required non-analytic scaling equations valid beyond the PAD are formulated using the effective power law forms $F_{P, e \text {, th }}(t)=\Pi_{P, e, \text { th }}^{ \pm}(t) t^{-\pi_{P, e \text { th }}(t)}$ of the mean crossover functions, where $\pi_{P, e, \mathrm{th}}(t)=-\frac{\partial \operatorname{Ln}\left[F_{P, \mathrm{th}}(t)\right]}{\partial \operatorname{Ln} t}$ is the effective exponent [33] and $\Pi_{P, e, \text { th }}^{ \pm}(t)$ is the related effective amplitude. Hence, the main goal of our present description performed beyond the PAD is the non-analytical and unequivocal scaling computation of the non-universal quantities, noted $\vartheta_{\mathcal{L}}^{ \pm}$, as functions of $\left|\Delta \tau^{*}\right|$ above $(+)$ and below $(-) T_{c} \cdot \vartheta_{\mathcal{L}}^{ \pm}$ are introduced through the linear forms $t=\vartheta_{\mathcal{L}}^{ \pm}\left|\Delta \tau^{*}\right|$, where $t$ is still the temperature-like field of the $\left(\phi^{2}\right)_{d=3}^{2}(n=1)$ model. However, as $\Delta \tau^{*}$ corresponds to a finite temperature range such that $\left|\Delta \tau^{*}\right|>\mathcal{L}_{\text {PAD }}^{f}$, we have no reason to assume that identities $\vartheta_{\mathcal{L}}^{+}=\vartheta_{\mathcal{L}}^{-} \equiv$ $\vartheta$ will be satisfied. In contrast, the local values of $\vartheta_{\mathcal{L}}^{ \pm}\left(\left|\Delta \tau^{*}\right|\right)$ appear to be numerically correlated to the local estimates of the theoretical pairs $\left\{\Pi_{P, \text { th }}^{ \pm} ; \pi_{P, e, \text { th }}\right\}$, only using the generalized critical coordinates of the vapor-liquid critical point. We can then find $\vartheta_{\mathcal{L}}^{ \pm}$from the tangent envelop of any continuous function $f_{P}\left(\left|\Delta \tau^{*}\right|\right)$ that fits the data measurements of the singular property $P_{s \text {, expt }}^{*}$ over a limited temperature range $\Delta \tau_{\min }^{*} \leq\left|\Delta \tau^{*}\right| \leq \Delta \tau_{\max }^{*}$ such as $\Delta \tau_{\text {min }}^{*}>\mathcal{L}_{\mathrm{PAD}}^{f}$ and $\Delta \tau_{\text {max }}^{*} \sim \mathcal{L}_{\mathrm{EAD}}^{f}$. The conformity with the asymptotic Isinglike universal features calculated in the MR scheme of the $\left(\phi^{2}\right)_{d=3}^{2}(n=1)$ model [3,5] is then maintained. This local characterization can thus be formulated to describe the fluid data away from the immediate vicinity of the critical point. That means that our additional non-analytical scaling equations can be useful in a temperature range $\Delta \tau^{*}>\mathcal{L}_{\mathrm{PAD}}^{f}$ where the deviations from Eq. (1) and the measured fluid properties become significant. This goal will be schematically illustrated below for the susceptibility case, through Fig. 1, where the inserted part also puts in evidence the meaningfull digits needed for accurate estimations of the non-universal parameters. Such a determination of the three non-universal parameters is then essentially different (while the extracted final results are similar) from the initial adjustement of fluid-dependent free parameters performed in Ref. [16] by a minimization of fitting errors over the experimental temperature ranges. Moreover, our present method provides the quantitative criteria to descriminate the physical conclusions which can be drawn about the fluid experiments performed either within the PAD or beyond the PAD, extending then the approach given in Ref. [21] for the xenon susceptibility case.

To illustrate our approach that strictly avoids adjusting the fluid-dependent free parameter $\vartheta_{\mathcal{L}}^{ \pm}$, xenon is used as a typical example of a (non-quantum) simple fluid, due to the extensive and accurate experimental data available along its critical isochore. Indeed, the two functions $f_{P}\left(\left|\Delta \tau^{*}\right|\right)$ of present concern are: (i) the very precise fitting results of Güttinger and Cannell [20] for the susceptibility data in the homogeneous domain $\left(T>T_{c}\right)$ and (ii) the very precise fitting results of Närger and Balzarini [34] for the symmetrical order parameter density data in the non-homogeneous domain $\left(T<T_{c}\right)$. In complement, we note that the observed non deviation from the rectilinear density diameter [34-36] and the high-symmetry nature of 
the experimental thermodynamic properties of xenon [35,37] avoid having to consider the effects of odd terms and mixing fields introduced in an effective scaling theory $[1,38]$. That allows us to verify the uniqueness of the $\vartheta_{\mathcal{L}}^{ \pm}$values and its exact matching with $\vartheta$.

The paper is organized as follows. Section 2 gives a brief analysis of the Ising-like features controlled by the ad hoc construction of the MR mean crossover functions given in Ref. [9]. The local three-parameter characterization of a simple fluid beyond the Ising-like preasymptotic domain is then schematically illustrated in Sect. 3 using the isothermal compressibility case above $T_{c}$. The non-analytic equations to calculate the local values of the crossover parameter $\vartheta_{\mathcal{L}}^{ \pm}$are provided in Sect. 4 and numerically solved analyzing the precise experimental fitting results for the isothermal compressibility case above $T_{c}$ and the order parameter density case below $T_{c}$ in the xenon case. Two remarks and a conclusion are given in Sect. 5. For convenience, Appendix 1 recalls the useful notations, definitions and expected estimations of the amplitudes and scale factors corresponding to the MR three-parameter characterization of any one-component fluid within the Ising-like preasymptotic domain. Special attention is given to demonstrate that these estimations only use four well-defined critical coordinates of the vapor-liquid critical point while the main literature sources in xenon case are used to support the needed numerical estimates. Appendix 2 gives an illustration of the non-analytical scaling determination of the very-precise local value of the crossover parameter $\vartheta_{\mathcal{L}}^{+}$, based on the effective pure power law $f_{\kappa_{T}}\left(\Delta \tau^{*}\right)=\Gamma_{e}^{+}\left(\Delta \tau^{*}\right)^{-\gamma_{e}}$ of the isothermal compressibility, noting that such effective power laws were commonly used in the 80 's to represent the fluid's singular behavior in a restricted temperature range.

\section{Ising-Like Characterization of a Simple Fluid Using the MR Crossover Functions}

\subsection{Distinction Between the Ising-Like Preasymptotic Domain and the Ising-Like Extended Asymptotic Domain}

As explained in detail in Ref. [9], the dimensionless ad hoc construction of the MR mean crossover functions provides the best control of the error-bar amplitude and propagation of their related min and max estimations given in Ref. [5], when approaching the non-trivial fixed point. The resulting mean crossover functions are thus written in the following convenient form

$$
P_{\text {th }}(t)=\mathbb{Z}_{P}^{ \pm} t^{-e_{P}} \Pi_{P, \text { th }}\left(t^{D(t)}\right)
$$

where the following well-defined two-term Wegner-like expansion

$$
P_{\mathrm{PAD}, \mathrm{th}}(t)=\mathbb{Z}_{P}^{ \pm} t^{-e_{P}}\left(1+\mathbb{Z}_{P}^{1, \pm} t^{\Delta}\right)
$$

characterizes the universal Ising features estimated in the asymptotical Ising-like limit $t \rightarrow 0$. Indeed, in Eq. (2), each function $\Pi_{P, \text { th }}\left(t^{D(t)}\right)$ is given in the form of a three-term product of the variable $t^{D(t)}$. The exponent function $D(t)=\frac{\Delta_{\mathrm{MF}} S_{2} \sqrt{t}+\Delta}{S_{2} \sqrt{t}+1}$ is independent of $P_{\mathrm{th}}$ and expresses the crossover of the effective confluent exponent which varies between $\Delta$ and $\Delta_{\mathrm{MF}}$ in the complete range $t=\{0, \infty\}$. It is then possible to define a single theoretical crossover temperature $t_{\Delta}$ (noted $t_{0}^{*}$ in [9]) given by

$$
t_{\Delta} \simeq\left(\frac{1}{S_{2}}\right)^{2} \cong 1.9 \times 10^{-3}
$$


where $D(t)$ takes the effective mean value $D\left(t_{\Delta}\right)=\frac{\Delta+\Delta_{\mathrm{MF}}}{2}$. Consequently, $t_{\Delta}$ characterizes the exchange between the prominent Ising-like crossover nature due to $\Delta=0.50189$ when approaching the non-trivial fixed point $\left[t \ll t_{\Delta}\right]$, to the prominent mean field-like crossover nature due to $\Delta_{\mathrm{MF}}=\frac{1}{2}$ when approaching the trivial fixed point $\left[t \gg t_{\Delta}\right]$, along the renormalized trajectory.

In the following, we are only interested in the Ising-like universal domain $t \leq t_{\Delta}$ since we have already noted that the Ising-like critical crossover in one-component fluids stops before they display a true classical behavior certainly not accounted for by the classical behavior governed by the trivial fixed point in field theory. More precisely, it was introduced in [9] the following common temperaturelike borderline

$$
\mathcal{L}_{\mathrm{PAD}}^{\mathrm{Ising}}=\varpi t_{\Delta} \simeq 1.9 \times 10^{-6}
$$

with $\varpi \cong 10^{-3}$, which distinguishes, either the Ising-like preasymptotic domain $t \lesssim \varpi t_{\Delta}$ where the restricted Eq. (3) is valid, or the intermediate Ising-like crossover domain $\varpi t_{\Delta}<$ $t<t_{\Delta}$ where it is needed to consider the complete Eq. (2). In the latter case, Eq. (2) can also account, in a practical manner (see below Sect. 3), for many sources of nonuniversality discarded or assumed small in the MR scheme because they are unessential in the vicinity of the critical point.

\subsection{Brief Recall of the Expected PAD Description}

All the supporting materials involved in the expected PAD description using Eq. (3) were already published and mainly summarized in Ref. [11] for any one-component fluid $f$ whose generalized (four) critical point coordinates are known. For self-consistent lecture of the present paper, they are recalled in Appendix 1.

Hereafter we shall consider three theoretical crossover functions $F_{P, \text { th }}(t, h=0)$ defined in Ref. [9], namely, the dimensionless correlation length $\ell_{\text {th }}(t)$ and the dimensionless magneticlike susceptibility $\chi_{\mathrm{th}}(t)$ above $T_{c}$, and the dimensionless magnetizationlike density $m_{t h}(|t|)$ below $T_{c} . t$ is the theoretical temperaturelike field and $h=0$ is the zero value of the external ordering (magneticlike) field. Selecting these three functions guaranties that the same single length unit is used to reduce the correlation and thermodynamics properties since the dimensionless quantity $\left(\chi_{\mathrm{th}}\right)^{-1}\left(m_{t h}\right)^{2}\left(\ell_{\mathrm{th}}\right)^{3}$ leads to the universal amplitude combination $Q_{c}$ for the critical point limit $|t| \rightarrow 0$.

The theoretical functions are then used for analyzing three (Ising-like-similar) fluid properties along the critical isochore $(\Delta \tilde{\rho}=0)$ of a one-component fluid (labelled $f$ ) with a vanishing external (magnetic-like) field $(\Delta \tilde{\mu}=0) . \Delta \tilde{\rho}=\frac{\rho-\rho_{c}}{\rho_{c}}$ is the reduced order parameter density and $\Delta \tilde{\mu}=\tilde{\mu}-\tilde{\mu}_{c}$ is the conjugated ordering field, with $\tilde{\mu}=\frac{\mu_{\rho} \rho_{c}}{p_{c}}\left(\tilde{\mu}_{c}=\frac{\mu_{\rho, c} \rho_{c}}{p_{c}}\right)$. $\rho$ is the mass density and $\mu_{\rho}$ is the conjugated chemical potential per mass unit. The subscript $\rho$ refers to a specific (per mass unit) quantity. The subscript $c$ refers to a critical parameter of $f$, such as the critical pressure $p_{c}$. The distance to the vapor-liquid critical point is then only defined by the reduced temperature difference $\Delta \tau^{*}=\frac{T-T_{c}}{T_{c}}$ where $T$ is the temperature and $T_{c}$ the critical temperature of $f$. The selected singular properties are the dimensionless correlation length $\xi_{\text {expt }}^{*}\left(\Delta \tau^{*}\right)$, the dimensionless isothermal compressibility $\kappa_{T \text {,expt }}^{*}\left(\Delta \tau^{*}\right)$ in the homogeneous domain $\Delta \tau^{*}>0$, and the spontaneous non-zero value of the (symmetrical) dimensionless order parameter density $\Delta \tilde{\rho}_{L V \text {, expt }}\left(\left|\Delta \tau^{*}\right|\right)$ in the non-homogeneous domain $\Delta \tau^{*}<0$. In this fluid case, the dimensional correlation length $\xi$ corresponds to the correlation length that is obtained by considering the small-momentum behavior of the structure factor, while the normalized thermodynamic description of $f$ in the grand 
canonical ensemble considers the quantities per particle (using thus the subscript $\bar{p}$ ). The dimensionless quantity $\beta\left(\chi_{T, \bar{p}}\right)^{-1}\left(\Delta n_{L V}\right)^{2} \xi^{3}$ is then the analog of $\left(\chi_{\mathrm{th}}\right)^{-1}\left(m_{t h}\right)^{2}\left(\ell_{\mathrm{th}}\right)^{3}$, where $\beta$ is the inverse of an energy reference, $\chi_{T, \bar{p}}=\left(\frac{\partial n}{\partial \mu_{\bar{p}}}\right)_{T}$ is the isothermal susceptibility per particle, and $\Delta n_{L V}=n_{L}-n_{V}$ is the difference in number density of particles between the liquid and vapor coexisting phases below $T_{c} . n$ is the number density, with $n m_{\bar{p}}=\rho$ and $\frac{\mu_{\bar{p}}}{m_{\bar{p}}}=\mu_{\rho}$, where $m_{\bar{p}}$ is the (molecular) mass of the particle. We recall that $\chi_{T, \bar{p}}=\left(m_{\bar{p}}\right)^{-2} \chi_{T, \rho}=n^{2} \kappa_{T}$, where $\chi_{T, \rho}=\left(\frac{\partial \rho}{\partial \mu_{\rho}}\right)_{T}$ is the isothermal susceptibility per mass unit and $\kappa_{T}=\frac{1}{n}\left(\frac{\partial n}{\partial p}\right)_{T}=\frac{1}{\rho}\left(\frac{\partial \rho}{\partial p}\right)_{T}$ is the isothermal compressibility. The corresponding dimensionless quantities $\xi^{*}$ and $\kappa_{T}^{*}$ are obtained using the length $\left(\alpha_{c}=\left(\frac{k_{B} T_{c}}{p_{c}}\right)^{\frac{1}{3}}\right)$ and energy $\left(\left(\beta_{c}\right)^{-1}=k_{B} T_{c}\right)$ units, while $\Delta \tilde{\rho}_{L V}=\Delta n_{L V} \frac{m_{\bar{p}}}{\rho_{c}}$.

Therefore, the characterization of the Ising-like PAD description takes the set forms $\mathbb{S}_{A}^{\{M R\}}=\left\{\mathbb{Z}_{\chi}^{1,+} ;\left(\mathbb{Z}_{\xi}^{+}\right)^{-1} ;\left(\mathbb{Z}_{\chi}^{+}\right)^{-1}\right\}, \mathcal{S}_{A}^{\{1 f\}}=\left\{\mathcal{Z}_{\chi}^{1,+} ; \mathcal{Z}_{\xi}^{+} ; \mathcal{Z}_{\chi}^{+}\right\}$, and $S_{A, f}=\left\{a_{\chi}^{+} ; \xi^{+} ; \Gamma^{+}\right\}$ made of three independent amplitudes. Each set (where subscript $A$ recalls the amplitude nature of the involved parameters) characterizes, successively, the theoretical MR mean crossover functions for the $O(1)$ universality class, the master crossover functions for the $\{1 f\}$ universality subclass and, finally, any one-component fluid $f$. This expected Ising-like PAD description only requires the knowledge of the location of the vapor-liquid critical point in the experimental phase surface of $f$. Indeed, the four critical coordinates of $f$ are used to define: (i) the energy $\beta_{c}$ and length $\alpha_{c}$ units involved in the dimensionless reduction process of the thermodynamics properties and the correlation length and, (ii) the two dimensionless critical numbers $Y_{c}$ and $Z_{c}$ which are respectively the characteristic scale parameters of the relevant fields along the critical isochore and the critical isotherm of $f$. That defines the three non-universal parameter set $S_{S F, f}^{\{1 f\}}=\left\{Y_{c} ; \alpha_{c} ; Z_{c}\right\}$ (where subscript $S F$ recalls the scale factor nature of the involved parameters) of each fluid $f$ belonging to the $\{1 f\}$ universality subclass [28-30]. On the other hand, the three non-universal scale factor set $S_{S F, f}^{\{M R\}}=\left\{\vartheta ; g_{0} ; \psi_{\rho}\right\}$ of each fluid $f$ belonging to the $O$ (1) universality subclass are thus introduced in the mean crossover functions under the form of two characteristic scale factors $\left(\vartheta\right.$ and $\left.\psi_{\rho}\right)$ and a single coupling constant $\left(g_{0}\right)$. The latter $g_{0}$ has the convenient wavelength dimension at $d=3$ to take the practical role of a finite wavelength cutoff, defined at the vapor-liquid critical point [16]. $\vartheta$ is defined by the asymptotical linearized approximation $t=\vartheta \Delta \tau^{*}$ when $t \rightarrow 0$ and $\Delta \tau^{*} \rightarrow 0$ [6] along the critical isochore of $f . \psi_{\rho}$ is associated to the Ising-like asymptotic singular behaviors along the critical isotherm of $f$, by using the similar asymptotical linearized approximation when the intensive fields $h \rightarrow 0$ and $\Delta \tilde{\mu} \rightarrow 0$ or, equivalently, the conjugated densities $m_{\text {th }} \rightarrow 0$ and $\Delta \tilde{\rho} \rightarrow 0$. The inverse wavelength $\left(g_{0}\right)^{-1}$ is the natural microscopic length unit for the comparison of $\ell_{\text {th }}(t)$ to the dimensional correlation length $\xi_{\exp }\left(\Delta \tau^{*}\right)$, through the fitting equation $\ell_{\mathrm{PAD} \text {,th }}(t)=g_{0} \xi_{\text {expt }}\left(\Delta \tau^{*}\right)$. The estimation of $\vartheta, \psi_{\rho}$, and $g_{0}$ are made without any adjustable parameter when the four critical coordinates of $f$ are known. These determinations take advantage of the Ising-like master singular behavior of the one-component fluid subclass described by the master crossover functions defined in Ref. [11], introducing the three-master scaling constant set $\mathcal{S}_{S C}^{\{1 f\}}=\left\{\Theta^{\{1 f\}} ; \mathbb{L}^{\{1 f\}} ; \Psi^{\{1 f\}}\right\}$ (where subscript $S C$ recalls for scaling constants), which links the corresponding Ising-like fields. As a result, the unequivocal relations between the scale factor sets $S_{S F, f}^{\{M R\}}=\left\{\vartheta ; g_{0} ; \psi_{\rho}\right\}$ and $S_{S F, f}^{\{1 f\}}=\left\{Y_{c} ; \alpha_{c} ; Z_{c}\right\}$, and 
finally the amplitude set $S_{A, f}=\left\{a_{\chi}^{+} ; \xi^{+} ; \Gamma^{+}\right\}$can be easilly written, noting that the following master constant $\mathbb{L}^{\{1 f\}}=g_{0} \alpha_{c}=25.585$ guaranties the uniqueness of the length unit. Consequently, the complete fluid sets of leading amplitudes [such as $\Pi_{P}^{ \pm}$in Eq. (1)] and confluent amplitudes (such as $a_{P}^{ \pm}$in Eq. (1)), can be calculated using $\vartheta, \psi_{\rho}$, and $g_{0}$. The resulting universal asymptotic Ising-like features (including the universal features related to the first order confluent corrections) of the $\{1 f\}$ universality subclass are thus exactly conform with the ones of the $O(1)$ universality class, as recalled in Appendix 1.

Here, introducing the complete theoretical set of the universal confluent amplitudes $\mathbb{Z}_{P}^{1, \pm}$ involved in the MR mean crossover functions, it is essential to underline the following unequivocal scaling equations which characterize the PAD description

$$
\vartheta=\left(\frac{a_{P}^{ \pm}}{\mathbb{Z}_{P}^{1, \pm}}\right)^{\frac{1}{\Delta}}
$$

whatever the selected $P$. Indeed, Eq. (6) demonstrates that the single fluid scale factor $\vartheta$ characterizes the first order contribution of the lowest confluent corrections to scaling to be conform with the corresponding Ising-like universal ratios estimated in the MR scheme. In addition, the knowledge of $\vartheta$ also infers the unequivocal estimation of

$$
\mathcal{L}_{\mathrm{PAD}}^{f}=\frac{\mathcal{L}_{\mathrm{PAD}}^{\mathrm{Ising}}}{\vartheta(f)}
$$

which means that the two-term singular behaviors, as well as their validity temperature range, are well estimated. Similarly, as the dimensionless correlation length $\xi_{\text {expt }}^{*}=\frac{\xi_{\text {expt }}}{\alpha_{c}}$ for $f$ is necessarily expressed in unit of the size $\alpha_{c}$ of the critical interaction cell [28], the introduction of $\mathbb{L}^{\{1 f\}}=g_{0} \alpha_{c}$ leads to the following convenient form of the fitting equation

$$
\frac{\ell_{\mathrm{th}}(t)}{\mathbb{L}^{\{1 f\}}} \equiv \frac{\xi_{\text {expt }}\left(\Delta \tau^{*}\right)}{\alpha_{c}}
$$

Equation (8) is a direct estimation of the ratio between long-range critical fluctuations and short-range microscopic interactions in $f$, i.e., a key parameter for distinguishing the critical and regular crossover regimes. Using the MR mean crossover function of the correlation length given in [9] and the above value $\mathcal{L}_{\mathrm{PAD}}^{\text {Ising }}$ of Eq. $(5), \ell_{\mathrm{th}}\left(\varpi t_{\Delta}\right)=1916$. Therefore, the Ising-like preasymptotic domain of interest corresponds to $\ell_{\text {th }} \gtrsim 1900$, i.e., $\frac{\xi_{\text {expt }}\left(\Delta \tau^{*}\right)}{\alpha_{c}} \geq$ 75 for any selected $f$. This large and increasing value of $\frac{\xi_{\text {expt }}\left(\Delta \tau^{*}\right)}{\alpha_{c}} \gg 1$ for $\Delta \tau^{*} \rightarrow 0$ confirms the expected dominant effect of the long-range critical fluctuations in the fluid PAD. Unfortunately, as mentionned in the introduction, there is no experimental data to validate with the required precision this expected PAD description, even fixing the critical exponents to their estimated theoretical values.

\subsection{MR Extended Asymptotic Domain Beyond the Ising-Like Preasymptotic Domain}

The main concrete progress to formulate a complete answer to the Ising-like crossover can be made analyzing another non-universal feature of the MR mean crossover functions. At the well-defined crossover temperature-like $t=t_{\Delta}$ of Eq. (4), $\ell_{\mathrm{th}}\left(t_{\Delta}\right)=28.8$ from [9], i.e., $\ell_{\text {th }}(t) \sim \mathbb{L}^{\{1 f\}}=25.585$. Therefore, the Ising-like crossover behavior where the theoretical confluent exponent function (which is property non-dependent) takes the effective mean 
value $\frac{\Delta+\Delta_{\mathrm{MF}}}{2}$, also corresponds to the condition $\ell_{\mathrm{th}}(t) \sim \mathbb{L}^{\{1 f\}}$. Accounting for the previous PAD extension $\ell_{\text {th }} \gtrsim \ell_{\text {th }}\left(\varpi t_{\Delta}\right)=1916$, we are then concerned by the use of Eq. (2) in the two-decade range $2000 \gtrsim \ell_{\text {th }} \gtrsim 20$, typically.

However, the estimation of the validity range of Eq. (2) can still be refined away from the non-trivial fixed point when its application is restricted to $f$. Indeed, from Eq. (8), the condition $\ell_{\text {th }}(t) \sim \mathbb{L}^{\{1 f\}} \simeq 25.585$ corresponds to the condition $\xi_{\exp }\left(\Delta \tau^{*}\right) \sim \alpha_{c}$, where the fluid correlation length is of the order of the size of the fluid critical interaction cell [28]. Accordingly, for any fluid case, the experimental condition $\xi_{\text {expt }}\left(\Delta \tau^{*}\right) \gtrsim \alpha_{c}$ can be well-understood as related to the Ising-like prominent nature of the crossover behavior for $\ell_{\mathrm{th}}(t) \geq \ell_{\mathrm{th}}\left(t_{\Delta}\right)$. However, our previous analyses of the validity range for the master singular behavior of the $\{1 f\}$-subclass have shown that the scaling singular behavior of correlation length of seven one-component fluids [39] can only be observed correctly for $\frac{\xi_{\text {exp }}}{\alpha_{c}} \gtrsim 3$, typically. From such a practical limiting condition, the equivalent extension $\ell_{\text {th }}(t) \gtrsim$ $(2.5-3) \times \ell_{\text {th }}\left(t_{\Delta}\right) \simeq 70-90$ can be defined for the MR theoretical correlation length range of interest. More precisely, the following discussion shows that the realistic limiting range $\ell_{\text {th }} \gtrsim 2.6 \ell_{\text {th }}\left(t_{\Delta}\right) \simeq 75$ can be chosen, leading to estimate the extension of the corresponding temperaturelike range of the Ising-like EAD

$$
t \leq \mathcal{L}_{\mathrm{EAD}}^{\mathrm{Ising}}=3.9 \times 10^{-4}
$$

Here Ising-like EAD means that practically a single scale factor can be used for the relevant field and the irrelevant field which are accounted for along this primary critical path. Using thus the asymptotic value of $\vartheta$ provided by our expected PAD description of $f$, the corresponding expected EAD extension for $f$ corresponds to

$$
\Delta \tau^{*} \leq \mathcal{L}_{\mathrm{EAD}}^{f}=\frac{\mathcal{L}_{\mathrm{EAD}}^{\text {Ising }}}{\vartheta}
$$

In addition, we remark that Eq. (8) provides a useful tool to express any singular thermodynamic property as a function of either $\Delta \tau^{*}$ or $\ell_{\text {th }}$ (as illustrated in figures below using the asymptotic value of $\vartheta$ calculated in Appendix 1 for the xenon case).

Now, the complete comparison between experimental results for $f$ and crossover theories can cover several decades above or below its $T_{c}$ value, but clearly distinguishing the physical expected description within the PAD from the unknown one beyond the PAD. In the latter case, the intrinsic difficulties associated with the usual fitting over a limited temperature range have been illustrated in the recent re-analysis of the critical xenon data [21] from the Earth experiments, performed by Güttinger and Cannell [20]. Simultaneously, we have noted that a relatively complete set of xenon leading amplitudes $\Pi_{P}^{ \pm}$exists in the litterature (see for example Tables 3 and $4^{1}$ in Ref. [21]), despite their extraction from measurements largely beyond the PAD. However, even for such extensive xenon description assumed conform with the two-scale-factor universality valid for the Ising-like asymptotic limit $\Delta \tau^{*} \rightarrow 0$, it remains challenging to carefully probe the uniqueness and understand the physical critical nature of the third parameter, characteristic of the confluent singular behavior at large distance away from $T_{c}$.

1 In Tables 2, and 4 of Ref. [21], the reported data in columns 4 and 5 are not correct since $\Delta$ is not used in related Ref. [50]. Therefore, n.u. should be used in column 4 and no value of $a_{\chi}^{1,+}$ should be reported in column 5 


\section{Fluid Characterization Beyond the Ising-Like Preasymptotic Domain}

\subsection{Fitting Equations Using the MR Mean Crossover Functions Beyond the PAD}

The description of the fluid singular property $P_{s, \exp }^{*}\left(\Delta \tau^{*}\right)$ in the finite temperature range such as $\Delta \tau^{*}>\mathcal{L}_{\mathrm{PAD}}^{f}$, generally uses a truncated Wegner expansion, which involves a finite serie of correction-to-scaling terms higher than the first leading one given in Eq. (1) [or Eq. (3)]. The validity of this truncated expansion can be observed a posteriori over the extended asymptotic domain defined by $\Delta \tau^{*} \leq \mathcal{L}_{\mathrm{EAD}}^{f}$. Unfortunately, ignoring Eq. (10), the resulting experimental extension $\mathcal{L}_{\text {EAD }}^{f}$ appears highly dependent on the number and the nature of the several correction-to-scaling terms selected in the finite serie. Such a truncated description in the finite intermediate range $\mathcal{L}_{\mathrm{PAD}}^{f}<\Delta \tau^{*} \leq \mathcal{L}_{\mathrm{EAD}}^{f}$ is not able to evaluate any theoretical estimate. The main reason is that we cannot solve new correlative difficulties concerning the effective number (which can thus be greater than three) and the actual nature (which can originate from different analytical and confluent effects in the theoretical schemes) of the fluid-dependent parameters.

Some of these difficulties to describe $P_{s, \exp }^{*}\left(\Delta \tau^{*}\right)$ can be by-passed by using the mean crossover function $F_{P, \text { th }}(t)$ defined in [9], despite the absence of information concerning the extended asymptotic domain of $f$ and the non-evaluation of the effective influence of the numerous corrections neglected in the massive renormalization scheme of the $\left(\phi^{2}\right)_{d=3}^{2}(n=1)$ model (see [9] for details). For this purpose, the fitting equation of $P_{s, \exp }^{*}\left(\Delta \tau^{*}\right)$ must then be written as follows $[5,11]$

$$
P_{s, \exp }^{*}\left(\Delta \tau^{*}\right)=\mathbb{P}_{0, \mathcal{L}^{*}} \mathbb{Z}_{P}\left(\Delta \tau^{*}\right)^{-e_{P}} \Pi_{P, \text { th }}\left(t^{D(t)}\right)
$$

where the inserted adjustable fluid-dependent parameters are the (property-dependent) prefactor $\mathbb{P}_{0, \mathcal{L}}^{*}$ and the crossover parameter $\vartheta_{\mathcal{L}}^{ \pm}$introduced through the relation:

$$
t=\vartheta_{\mathcal{L}}^{ \pm} \Delta \tau^{*}
$$

We note that the subscript $\mathcal{L}$ underlines the fact that the fitting determination of such a fluiddependent pair $\left\{\mathbb{P}_{0, \mathcal{L}}^{*}, \vartheta_{\mathcal{L}}\right\}$ is performed beyond the PAD. Anticipating the main difference with our non-analytical scaling method proposed below, we underline the fact that, in a joint fitting process of all the singular properties along the critical isochore, $\vartheta_{\mathcal{L}}$ is expected to have the same single value above or below $T_{c}$, i.e., $\vartheta_{\mathcal{L}}=\vartheta_{\mathcal{L}}^{+}=\vartheta_{\mathcal{L}}^{-}$. In Eq. (11), $F_{P \text {,th }}(t)$ is now splitted in a leading power law term $\mathbb{P}_{0, \mathcal{L}^{*}} \mathbb{Z}_{P}\left(\Delta \tau^{*}\right)^{-\pi_{P}}$, only expressed as a function of the physical temperature field $\Delta \tau^{*}$, and in a confluent function $\Pi_{P, \text { th }}\left(t^{D(t)}\right)$, only dependent on $\vartheta_{\mathcal{L}}^{ \pm}$, through Eq. (12). The fluid-dependent, property-dependent prefactor $\mathbb{P}_{0, \mathcal{L}}^{*}$ accounts for the interpolated asymptotic singular behavior of $P_{\exp }^{*}$ for $\Delta \tau^{*} \rightarrow 0$, while the resulting fitting value $\vartheta_{\mathcal{L}}=\vartheta_{\mathcal{L}}^{+}=\vartheta_{\mathcal{L}}^{-}$is a non-universal parameter which characterizes the finite intermediate range $\mathcal{L}_{\mathrm{PAD}}^{f}<\Delta \tau^{*} \leq \mathcal{L}_{\mathrm{EAD}}^{f}$, as mentioned in the Introduction. Here interpolated means that the above leading pure power law using $\mathbb{P}_{0, \mathcal{L}}^{*}$ as a free parameter cannot directly be supported by (non-available) experimental measurements within the PAD.

Among the prefactors $\mathbb{P}_{0, \mathcal{L}}^{*}$ involved in Eq. (11), only two (noted $\mathbb{L}_{0, \mathcal{L}}^{*}, \mathbb{X}_{0, \mathcal{L}}^{*}$ in next section), are independent and selected as two characteristic asymptotic parameters of $f$ to be conform with the two-scale-factor universality. In a fitting process where the identities $\vartheta_{\mathcal{L}}=\vartheta_{\mathcal{L}}^{+}=\vartheta_{\mathcal{L}}^{-}$are assumed, $\vartheta_{\mathcal{L}}$ is the third characteristic crossover parameter of $f$, from 
which the expected upper limit of the fluid extended asymptotic domain can be defined from $\mathcal{L}_{\text {EAD }}^{f}=\frac{\mathcal{L}_{\text {EAD }}^{\text {Ising }}}{\vartheta_{\mathcal{L}}}$.

\subsection{Characteristic Physical Parameters Using MR Crossover Functions Beyond the PAD}

Accordingly with Eq. (11), the fitting equations of $\xi_{\text {expt }}^{*}, \kappa_{T \text {,expt }}^{*}$, and $\Delta \tilde{\rho}_{L V \text {,expt }}$ for a simple fluid are:

$$
\begin{aligned}
\xi_{\text {expt }}^{*}\left(\Delta \tau^{*}\right) & =\frac{\mathbb{L}_{0, \mathcal{L}}^{*}}{\mathbb{Z}_{\xi}^{+}\left(\Delta \tau^{*}\right)^{v} \Pi_{\xi}^{+}\left(t^{D(t)}\right)} \\
\kappa_{T, \text { expt }}^{*}\left(\Delta \tau^{*}\right) & =\frac{\mathbb{X}_{0, \mathcal{L}}^{*}}{\mathbb{Z}_{\chi}^{+}\left(\Delta \tau^{*}\right)^{\gamma} \Pi_{\chi}^{+}\left(t^{D(t)}\right)} \\
\Delta \tilde{\rho}_{L V, \text { expt }}\left(\left|\Delta \tau^{*}\right|\right) & =\mathbb{M}_{0, \mathcal{L}}^{*} \mathbb{Z}_{M}\left(\left|\Delta \tau^{*}\right|\right)^{\beta} \Pi_{M}\left(|t|^{D(|t|)}\right)
\end{aligned}
$$

where the Ising-like universal value of $Q_{c}$ must be recovered through the following prefactor combination

$$
\left(\mathbb{L}_{0, \mathcal{L}}^{*}\right)^{d} \frac{\left(\mathbb{M}_{0, \mathcal{L}}^{*}\right)^{2}}{\mathbb{X}_{0, \mathcal{L}}^{*}}=1
$$

From fitting Eqs. (13) and (14), the expected new three-parameter characteristic set of $f$ is

$$
S_{1 C P, \mathcal{L}, f}^{\{M R\}}=\left\{\vartheta_{\mathcal{L}} ; \mathbb{L}_{0, \mathcal{L}}^{*} ; \mathbb{X}_{0, \mathcal{L}}^{*}\right\}
$$

The above set replaces the characteristic set $S_{S F, f}^{\{M R\}}=\left\{\vartheta ; g_{0} ; \psi_{\rho}\right\}$ previously defined from the PAD description of $f$. Obviously, when the identity $\vartheta \equiv \vartheta_{\mathcal{L}}$ is assumed valid, unequivocal links can be obtained between $\left\{\vartheta ; \psi_{\rho}\right\}$ and $\left\{\mathbb{L}_{0, \mathcal{L}}^{*} ; \mathbb{X}_{0, \mathcal{L}}^{*}\right\}$ (see Appendix 1). In Eq. (17), the subscript $1 C P, \mathcal{L}, f$ recalls for the use of the single crossover parameter $\vartheta_{\mathcal{L}}$ for $f$ until the upper limit $\Delta \tau^{*}<\mathcal{L}_{\mathrm{EAD}}^{f}=\frac{\mathcal{L}_{\mathrm{EAD}}^{\text {Ising }}}{\vartheta_{\mathcal{L}}(f)}$, where the value of $\mathcal{L}_{\mathrm{EAD}}^{\text {Ising }}$ given by Eq. (9) remains to be confirmed from our following MR theoretical description of the Ising-like EAD.

Therefore, the noticeable asymptotic feature of Eqs. (13) to (15) appears in each leading term in which $\vartheta_{\mathcal{L}}$ is no longer involved in the asymptotic scaling part of the critical behavior expressed in terms of the physical field $\Delta \tau^{*}$. Moreover, $\mathbb{X}_{0, \mathcal{L}}^{*}$ and $\mathbb{L}_{0, \mathcal{L}}^{*}$ are two prefactors that characterize the asymptotic Ising-like nature of xenon, provided that all the non-dimensional extensive variables are expressed with the same length unit [7]. Correlatively, the same value of $\vartheta_{\mathcal{L}}$ above and below $T_{c}$ exclusively controls the magnitude of many correction terms to scaling. That means that $\vartheta_{\mathcal{L}}$ can also integrate some effects of the neglected terms linked to the supplementary confluent exponents (noted $\Delta_{2}, \Delta_{3}$, etc.) associated to additional irrelevant operators, accounting then for practical numerical approximations such as $\Delta_{2} \approx 2 \Delta, \Delta_{3} \approx$ $3 \Delta$, etc. $\vartheta_{\mathcal{L}}$ can also integrate the contributions of analytical powered terms $\Delta \tau^{*},\left(\Delta \tau^{*}\right)^{2}$, etc., accounting for approximations such as $2 \Delta \approx 1,4 \Delta \approx 2$, etc., when $\left(k_{B}\right) T$ replaces $\left(k_{B}\right) T_{c}$ in the energy unit and in the dimensionless form of the temperature distance to the critical temperature, or when we consider the Massieu forms of the singular thermodynamic potentials. The determination of $\vartheta_{\mathcal{L}}$ is then equivalent to the determination of the effective extension $\mathcal{L}_{\mathrm{EAD}}^{f}$. As suggested in Ref. [5], from fitting at least three singular properties along the critical isochore (among the correlation length, the susceptibility, the specific heat in the 
homogeneous and non homogeneous domains, and the coexisting density measurements in the non-homogeneous domain), one must verify the uniqueness of the $\vartheta_{\mathcal{L}}$ value.

To avoid this large fitting task, we can now use an alternative remarkable facet of the Ising-like universality of each mean crossover function $F_{P}(t)$. Indeed, beyond the Ising-like preasymptotic domain, we can introduce the effective universal behavior of its local exponent $\pi_{P, e \text {,th }}(t)=-\frac{\partial \operatorname{Ln}\left[F_{P, \text { th }}(t)\right]}{\partial \operatorname{Ln}(t)}$, as initially proposed by Kouvel and Fisher [33]. It is then immediate to asymptotically transform (only using $\mathbb{P}_{0, \mathcal{L}}^{*}$ ) each theoretical universal function $F_{P, e \text {,th }}\left(\pi_{P, e, \text { th }}\right)$ into its fluid asymptotic property $\Pi_{P}^{ \pm}\left(\pi_{P, \text { th }}\right)$ valid for the Ising limit $\pi_{P, \text { th }}$. Moreover, $t_{\Delta}$ is a convenient sensor for a relative comparison with the $t$-values of the criticalto-classical crossing temperatures, where the $\pi_{P, e \text {,th }}(t)$ functions cross their mean crossover values $\pi_{P, \frac{1}{2}}\left(t_{\pi_{P, \frac{1}{2}}}\right)=\frac{\pi_{P, \mathrm{th}}+\pi_{P, \mathrm{MF}}}{2}$ (see Fig. 4 in [9]). Considering for example the correlation length and susceptibility cases, $t_{v_{\frac{1}{2}}} \cong 3 \times 10^{-3}$ and $t_{\gamma_{\frac{1}{2}}} \cong 4 \times 10^{-3}$, respectively, with $\ell_{\text {th }}\left(t_{v_{\frac{1}{2}}}\right)=22.2$ and $\ell_{\text {th }}\left(t_{\gamma_{\frac{1}{2}}}\right)=18.8$. These latter values are of the same order as $\ell_{\mathrm{th}}\left(t_{\Delta}\right) \simeq 28.8$, as well as the dimensionless master length $\mathbb{L}^{\{1 f\}}=25.585$. Therefore, the Ising-like prominent nature of any function $F_{P, e \text {,th }}\left(\pi_{P, e, \text { th }}\right)$, defined by the condition $\pi_{P, e \text {,th }}(t) \subset\left\{\pi_{P}, \pi_{P, \frac{1}{2}}\right\}$, is similar to the Ising-like prominent nature of the confluent function $D(t)$ in the temperature-like range $t \lesssim t_{\Delta}$. The use of the crossover behavior calculated in the $\left\{F_{P, e, \text { th }} ; \pi_{P, e, \text { th }}\right\}$ diagrams permits to distinguish, in a self-consistent manner, the expected functional forms where the non-universal parameters $\left(\mathbb{P}_{0, \mathcal{L}}^{*}, \vartheta_{\mathcal{L}}^{ \pm}\right)$are involved in each PAD or EAD range. Such functional forms are illustrated in the next section using the $\left\{\mathbb{Z}_{\chi, e}^{+} ; \gamma_{e, \text { th }}\right\}$ diagram for the susceptibility case already considered in the Appendix 2 of Ref. [11]. We limit however our present interest to the direct functional links between the MR mean crossover function and the theoretical-Ising-like susceptibility of an hypothetic fluid $f$ satisfying the related Ising-like universal features.

\subsection{Functional Forms for the Isothermal Susceptibility Case Above $T_{c}$}

The estimated theoretical behaviors of the effective exponent $\gamma_{e, \text { th }}(t)$ and the effective amplitude $\mathbb{Z}_{\chi, e}^{+}(t)$ are obtained from the equations

$$
\begin{gathered}
\gamma_{e, \mathrm{th}}(t)=-\frac{\partial \operatorname{Ln}\left[\chi_{\mathrm{th}}(t)\right]}{\partial L n t} \\
\mathbb{Z}_{\chi, e}^{+}(t)=\frac{\chi_{\mathrm{th}}(t)}{t^{-\gamma_{e}}}
\end{gathered}
$$

where $\chi_{\text {th }}(t)$ is given in [9]. By eliminating explicit $t$ in Eqs. (18) and (19), over the complete range $\gamma_{\mathrm{MF}} \leq \gamma_{e \text {,th }}(t) \leq \gamma$, the theoretical crossover behavior results in the form of the universal (mixed red) upper curve of implicit equation $\mathbb{Z}_{\chi, e}^{+}\left(\gamma_{e, t h}\right)$, labeled $\Phi_{3}(1)-\mathrm{MR}$ in Fig. 1.

Similarly, from the MR PAD description given in Appendix 1, we are able to calculate the expected classical-to-critical crossover of any one-component fluid $f$ over the complete range $\gamma_{\mathrm{MF}} \leq \gamma_{e}\left(\Delta \tau^{*}\right) \leq \gamma$. Indeed, $\gamma_{e}\left(\Delta \tau^{*}\right)$ and $\Gamma_{e}^{+}\left(\Delta \tau^{*}\right)$ of $f$ can be obtained from the equations

$$
\gamma_{e}\left(\Delta \tau^{*}\right)=-\frac{\partial \operatorname{Ln}\left[\kappa_{T, M R}^{*}\left(\Delta \tau^{*}\right)\right]}{\partial \operatorname{Ln}\left(\Delta \tau^{*}\right)}
$$




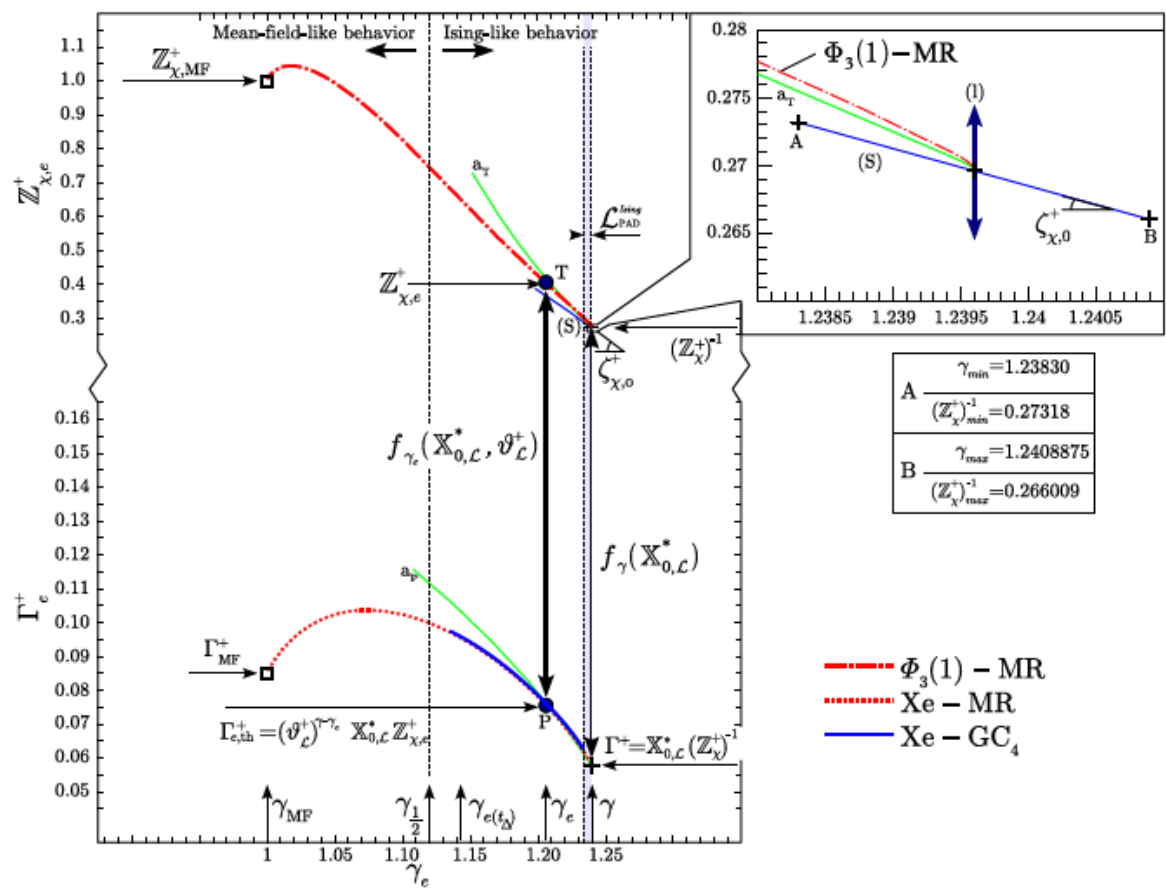

Fig. 1 Effective dimensionless amplitudes-exponent diagram for the susceptibility case. Upper mixed red curve labeled $\Phi_{3}(1)-$ MR : From $\chi_{\mathrm{th}}(t)$ given in [9], by using Eqs. (18) and (19). Lower dotted red curve labeled Xe - MR: From $\kappa_{T, M R}\left(\Delta \tau^{*}\right)$ by using Eqs. (20) and (21) and xenon physical parameters for the numerical construction. Lower full blue curve labeled Xe $-\mathrm{GC}_{4}:$ From $\kappa_{T \text {, expt }}\left(\Delta \tau^{*}\right)$ given by Eq. (25) [20], by using Eqs. (20) and (21). Vertical double arrows: two-parameter transformation $f_{\gamma_{e}}\left(\mathrm{X}_{0, \mathcal{L}}^{*}, \vartheta_{\mathcal{L}}^{+}\right)$from upper (T) and lower (P) points (full circles) at constant $\gamma_{e} \neq \gamma$ (see text and Appendix 2) and one-parameter transformation $f_{\gamma}\left(\mathbb{X}_{0, \mathcal{L}}^{*}\right)$ for the Ising-like limiting points (crosses) at $\gamma_{e} \equiv \gamma$. Curve labeled $a_{T}\left(a_{P}\right)$ : From Eq. (23) (From experimental case). Arrow $\gamma_{\frac{1}{2}}:$ mean value $\frac{\gamma+\gamma \mathrm{MF}}{2}$ related to the crossing temperature between the mean-field-like behavior $\left(\gamma_{\mathrm{MF}}<\gamma_{e}<\gamma_{\frac{1}{2}}\right)$ and the Ising-like behavior $\left(\gamma_{\frac{1}{2}}<\gamma_{e}<\gamma\right)$ (see also upper part and text). Insert : details of $\mathcal{L}_{\mathrm{PAD}}^{\text {Ising }}$ (see text and inserted Table). For other symbols and labels see text. See also Fig. 4 in Ref. [11] (Color figure online)

$$
\Gamma_{e}^{+}\left(\Delta \tau^{*}\right)=\frac{\kappa_{T, M R}^{*}\left(\Delta \tau^{*}\right)}{\left(\Delta \tau^{*}\right)^{-\gamma_{e}}}
$$

where we have hypothetized that $\kappa_{T, M R}^{*}\left(\Delta \tau^{*}\right)$ can be calculated using the corresponding Eq. (2) for $\chi_{\mathrm{th}}(t)$, introducing the expected scale factor set $S_{S F, f}^{\{M R\}}=\left\{\vartheta ; g_{0} ; \psi_{\rho}\right\}$ for $f$ given in Appendix 1. Eliminating $\Delta \tau^{*}$ (then also $\vartheta$ ), this expected "theoretical crossover behavior" for this hypothetic "Ising-like" $f$ corresponds to the lower curve (dotted red) in Fig. 1, of implicit equation $\Gamma_{e}^{+}\left(\gamma_{e}\right)$. This curve is here labeled $\mathrm{Xe}-\mathrm{MR}$ because we have used the xenon set $S_{S F, \mathrm{Xe}}^{\{M R\}}=\left\{\vartheta ; g_{0} ; \psi_{\rho}\right\}$ estimated in Table 1 of Appendix 1 for the corresponding numerical construction, without any restriction in our method when another one-component fluid is considered. Nevertheless, we note that our interest is restricted to the Ising-like range $\gamma_{e}$,th $(t) \geq \gamma_{\frac{1}{2}}=\frac{\gamma+\gamma_{\mathrm{MF}}}{2}$, as discussed above (in the upper part of Fig. 1, see the corresponding mean-field-like and Ising-like crossover behaviors separated by the mean value $\gamma_{\frac{1}{2}}=\frac{\gamma+\gamma_{\mathrm{MF}}}{2} \simeq 1.12$ ). 
Anticipating the matching (observed in the lower part of Fig. 1 and explained in next section) between the true experimental (full blue) curve $\Gamma_{e}^{+}\left(\gamma_{e, \text { expt }}\right)$ and the theoretical (dotted red) curve for $f$, it remains to define the related "physical" functional links illustrating the respective role of the two unknown fluid-dependent parameters $\mathbb{X}_{0, \mathcal{L}}^{*}$ and $\vartheta_{\mathcal{L}}^{+}$involved in fitting Eq. (14). We recall that $\vartheta_{\mathcal{L}}^{+}$must be introduced using the arbitrary relation

$$
t=\vartheta_{\mathcal{L}}^{+} \Delta \tau^{*}
$$

only considering the finite temperature range $\Delta \tau^{*}>0$ well beyond the PAD.

The first range $\gamma-\gamma_{e, \text { th }} \lesssim \mathbb{Z}_{\chi}^{1,+} \Delta\left(\mathcal{L}_{\mathrm{PAD}}^{\text {Ising }}\right)^{\Delta} \approx 0.006$ corresponds to the Ising-like preasymptotic domain (see the grey area in Fig. 1) where the experimental data are absent (or of limited precision). Then, this small domain can only be magnified in the theoretical insert of Fig. 1 for the close vicinity of the Ising-like limiting point of theoretical coordinates $\left\{\gamma ;\left(\mathbb{Z}_{\chi}^{+}\right)^{-1}\right\}$ (upper cross in Fig. 1). The curve $\mathrm{a}_{\mathrm{T}}$ corresponds to the asymptotic singular behavior of the derivative $\left(\frac{\partial \mathbb{Z}_{\chi, e}^{+}}{\partial \gamma_{e}}\right)_{\gamma_{e} \rightarrow \gamma}[11]$, of equation

$$
\begin{aligned}
\left(\frac{\partial \mathbb{Z}_{\chi, e}^{+}}{\partial \gamma_{e}}\right)_{\gamma_{e, \text { th } \rightarrow \gamma}=} & \left(\mathbb{Z}_{\chi}^{+}\right)^{-1}\left\{1+\left(\frac{\gamma-\gamma_{e, \text { th }}}{\Delta\left|\mathbb{Z}_{\chi}^{1,+}\right|}\right)^{-\left(\frac{\gamma-\gamma_{e, \text { th }}}{\Delta}\right)}\right. \\
& \left(1-\log \left[\frac{\gamma-\gamma_{e, \text { th }}}{\left.\left.\left.\Delta\left|\mathbb{Z}_{\chi}^{1,+}\right|\right]\right)\left(\frac{\gamma-\gamma_{e, \text { th }}}{\Delta}\right)\right\}}\right.\right.
\end{aligned}
$$

The vertical double arrow (1) indicates the logarithmic divergence of $\left(\frac{\partial \mathbb{Z}_{\chi, e}^{+}}{\partial \gamma_{e}}\right)_{\gamma_{e, t h} \rightarrow \gamma}$ never directly estimated from the asymptotic theoretical approaches (see also part (b) of Fig. 5 in Ref. [11]). We note the significant difference between the curve $\mathrm{a}_{\mathrm{T}}$ and the curve $(S)$. The latter one corresponds to the linearized slope $\zeta_{\chi, 0}^{+}=\frac{\left(\mathbb{Z}_{\chi, \max }^{+}\right)^{-1}-\left(\mathbb{Z}_{\chi, \min }^{+}\right)^{-1}}{\gamma_{\min }-\gamma_{\max }}=\frac{0.007171}{0.0025875} \simeq 2.8$ between the respective bounded coordinates of points A and B (see inserted table in Fig. 1 and Ref. [5] for the data sources). Indeed, the curve $(S)$ illustrates the analytical error-bar correlation between the Ising values of $\gamma$ and $\left(\mathbb{Z}_{\chi}^{+}\right)^{-1}$. Moreover, the magnified scales of the inserted part of Fig. 1 illustrate the fact that all the quoted digits in the present work are meaningfull to reveal this amazing theoretical asymptotic behavior.

Accordingly, the Ising-like limiting point of $f$ (xenon here, as explained just above), with coordinates $\left\{\gamma ; \Gamma^{+}=\mathbb{X}_{0, \mathcal{L}}^{*}\left(\mathbb{Z}_{\chi}^{+}\right)^{-1}\right\}$, corresponds to the ending lower cross of the curve Xe - MR. Similarly, the curve ap corresponds to the asymptotic singular behavior of $\left(\frac{\partial \Gamma_{e}^{+}}{\partial \gamma_{e}}\right)_{\gamma_{e} \rightarrow \gamma}$. The double arrow joigning the two crosses at $\gamma_{e}=\gamma$ schematizes the amplitude transformation $f_{\gamma}\left(\mathbb{X}_{0, \mathcal{L}}^{*}\right) \equiv \mathbb{X}_{0, \mathcal{L}}^{*}$ where $\vartheta_{\mathcal{L}}^{+}$has disappeared. $\mathbb{X}_{0, \mathcal{L}}^{*}$ only governs the universal matching of the Ising-like limiting point. Therefore, the introduction of the true leading value of $\Gamma^{+}$fixes the value of $\mathbb{X}_{0, \mathcal{L}}^{*}$ using the following Eq. [11]

$$
\begin{aligned}
\mathbb{X}_{0, \mathcal{L}}^{*} & =\left(\mathbb{L}^{\{1 f\}}\right)^{d}\left(\psi_{\rho}\right)^{2} \vartheta-\gamma \\
& =\left(\mathbb{L}^{\{1 f\}}\right)^{d}\left(\Psi^{\{1 f\}}\right)^{2}\left(\Theta^{\{1 f\}}\right)^{-\gamma}\left(Y_{c}\right)^{-\gamma}\left(Z_{c}\right)^{-1}
\end{aligned}
$$


On the top Eq. (24), the value of $\mathbb{X}_{0, \mathcal{L}}^{*}$ depends on the three asymptotic scale factors $\vartheta, \mathbb{L}^{\{1 f\}}$ (or $g_{0}$ ), and $\psi_{\rho}$. However, the master constant $\mathbb{L}^{\{1 f\}}$ fixes $g_{0}$, knowing $\alpha_{c}$. Therefore, $\vartheta$ and $\psi_{\rho}$ (the latter reflecting the extensive nature of the susceptibility) are the two characteristic parameters of $f$, in conformity with the two-scale factor universality. Similarly, in the bottom Eq. (24), we recall that $\Psi^{\{1 f\}}$ and $\Theta^{\{1 f\}}$ are the master constants, which guaranty that $Y_{c}$ and $Z_{c}$ are the two fluid-dependent parameters unequivocally linked to $\vartheta$ and $\psi_{\rho}$, respectively (see Appendix 1).

The comparison between the critical divergence of the initial slope (curves $\mathrm{a}_{\mathrm{T}}$ and ap) at the ending points provides the second characteristic constraint of the Ising-like preasymptotic domain. In principle, this second constraint must provide the unambiguous determination of the Ising-like scale factor $\vartheta$ [through $\vartheta=\left(\frac{a_{\chi}^{+}}{\mathbb{Z}_{\chi}^{1,+}}\right)^{\frac{1}{\Delta}}$, expressing Eq. (6) for $\chi$ ]. Nevertheless, our present geometrical description of the Ising-like preasymptotic domain given in the insert of Fig. 1 underlines the theoretical (and a fortiori experimental) challenging difficulties to provide the precise asymptotic characterization of the correction-to-scaling when a property reaches the Ising-like limiting point along a curve of universal, but infinite, slope for any Ising-like system (see also the discussion of the needed singular increase of the required precision in the last part of Appendix 2).

In the second range $0.12 \gtrsim \gamma-\gamma_{e} \gtrsim 0.015$, which corresponds to a temperature range largely beyond the Ising-like preasymptotic domain, the expected variations of $\mathbb{Z}_{\chi, e}^{+}\left(\gamma_{e, t h}\right)$ and $\Gamma_{e}^{+}\left(\gamma_{e}\right)$ are smooth and continuous. That implies that the fluid isothermal compressibility data obtained in the restricted temperature range $\Delta \tau_{\text {min }}^{*} \leq \Delta \tau^{*} \leq \Delta \tau_{\text {max }}^{*}$ can be fitted by any continuous function $\kappa_{T \text {,expt }}^{*}=f_{\kappa_{T}}\left(\Delta \tau^{*}\right)$. Applying then Eqs. (20) and (21), we expect to find at least one physical point $\mathrm{P}$ having the coordinates $\left\{\gamma_{e \text {,expt }} ; \Gamma_{e \text {,expt }}^{+}\right\}$in Fig. 1. Its matching condition with the lower curve $\mathrm{Xe}-\mathrm{MR}$ and its relation with the corresponding theoretical point $\mathrm{T}$ in the upper curve $\Phi_{3}(1)-\mathrm{MR}$ can be analyzed in terms of the two free parameters $\mathbb{X}_{0, \mathcal{L}}^{*}$ and $\vartheta_{\mathcal{L}}^{+}$. For the condition $\gamma_{e, \text { th }}(t)=\gamma_{e, \text { expt }}\left(\Delta \tau^{*}\right)$, a two parameter transformation $f_{\gamma_{e}}\left(\mathbb{X}_{0, \mathcal{L}}^{*}, \vartheta_{\mathcal{L}}^{+}\right)$, schematized by a double arrow in Fig. 1, insures the correspondence between $\mathrm{T}$ and $\mathrm{P}$. The definition $f_{\gamma_{e}}\left(\mathbb{X}_{0, \mathcal{L}}^{*}, \vartheta_{\mathcal{L}}^{+}\right)$is given in the next Section from an experimental restricted Wegner-like expansion while Appendix 2 explains the use of an experimental effective single power law in xenon case.

\section{Non-Analytical Scaling Determination of the Ising-Like Crossover Parameter: Xenon Case as a Typical Example}

\subsection{The Isothermal Susceptibility Case Above $T_{c}$}

To find the explicit form of $f_{\gamma_{e}}\left(\mathbb{X}_{0, \mathcal{L}}^{*}, \vartheta_{\mathcal{L}}^{+}\right)$, we use the Güttinger and Cannell's susceptibility data of xenon [20] fitted by the following (four terms) Wegner-like expansion (labeled $\mathrm{GC}_{4}$ )

$$
\begin{aligned}
& \kappa_{T, \text { expt }}^{*}\left(\Delta \tau^{*}\right)=\Gamma^{+}\left(\Delta \tau^{*}\right)^{-\gamma}\left[1+a_{1 \chi}^{+}\left(\Delta \tau^{*}\right)^{\Delta}+\right. \\
&\left.a_{2 \chi}^{+}\left(\Delta \tau^{*}\right)^{2 \Delta}+a_{3 \chi}^{+}\left(\Delta \tau^{*}\right)^{3 \Delta}\right]
\end{aligned}
$$

Equation (25) is valid over the reduced temperature range $\Delta \tau_{\text {min }}^{*} \leq \Delta \tau^{*} \leq \Delta \tau_{\text {max }}^{*}$, with $\Delta \tau_{\text {min }}^{*}=9.115 \times 10^{-5}$ and $\Delta \tau_{\text {max }}^{*}=1.95 \times 10^{-2}$. The critical exponents $\gamma=1.241$ and $\Delta=$ 
0.496 were fixed to the theoretical values calculated at the time by Le Guillou and Zinn-Justin [40] from the renormalization-group approach. The values of the adjustable parameters were $\Gamma^{+}=0.0577( \pm 0.0001), a_{1 \chi}^{+}=1.29( \pm 0.03), a_{2 \chi}^{+}=-1.55( \pm 0.2), a_{3 \chi}^{+}=1.9( \pm 0.5)$ (the error bars quoted are one standard deviation allowing for the correlation between parameters, with an uncertainty of $\pm 0.5 \mathrm{mK}$ on the $T_{c}$ value, see Appendix 2). Eliminating then the variable $\Delta \tau^{*}$ by the combination of Eqs. (20) and (21) where $\kappa_{T}^{*}$ is given by Eq. (25), we obtain the (full blue) curve $\Gamma_{e}^{+}\left(\gamma_{e}\right.$,expt $)$ labeled $\mathrm{Xe}-\mathrm{GC}_{4}$ in the lower part of Fig. 1. The matching with the curve $\mathrm{Xe}-\mathrm{MR}$ is noticeable over the complete experimental range. That confirms that the transformation $f_{\gamma_{e}}\left(\mathbb{X}_{0, \mathcal{L}}^{*}, \vartheta_{\mathcal{L}}^{+}\right)$must contain both constraints needed to satisfy the (point) position and the related (tangent) direction. Therefore, the scaling nature of the crossover matching beyond the Ising-like preasymptotic domain is significantly different in the non-analytical scaling procedure which either eliminates or accounts for the

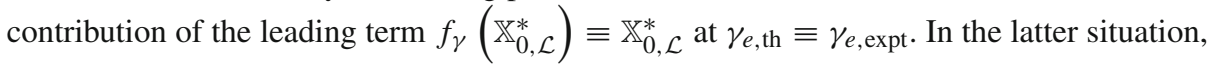
$\mathbb{X}_{0, \mathcal{L}}^{*}$ can be replaced by its estimate in term of the true leading amplitude $\Gamma^{+}$, as seen below.

In the first case where the contribution of the leading term is ignored, Eq. (14) is mainly equivalent to a prominant constraint in position, which can be expressed by the following relation between the effective exponents

$$
\gamma_{e, \operatorname{expt}}\left(\Delta \tau^{*}\right) \equiv \gamma_{e, \text { th }}\left[\vartheta_{\mathcal{L}}^{+}\left(\Delta \tau^{*}\right)\right]
$$

We numerically solve Eq. (26), using Güttinger and Cannell's fitting results given by Eq. (25), then providing the $\gamma_{e}\left(\Delta \tau^{*}\right)$ and $\vartheta_{\mathcal{L}}^{+}\left(\Delta \tau^{*}\right)$ values as functions of $\Delta \tau^{*}$. Both results are shown by the (full blue) curve labeled $\mathrm{GC}_{4}$ in Fig. $2 \mathrm{a}\left[\gamma_{e}\right.$ as a function of $\Delta \tau^{*}$ ], and the (full green) curve labeled 1 in Fig. $2 \mathrm{~b}\left[\vartheta_{\mathcal{L}}^{+}\right.$as a function of $\left.\Delta \tau^{*}\right]$, respectively. In part (a), we note that the experimental curve matches the (full red) curve labeled MR, which was obtained from Eq. (18) and $S_{S F, \mathrm{Xe}}^{\{M R\}}=\left\{\vartheta ; g_{0} ; \psi_{\rho}\right\}$ values given in Table 1 of Appendix 1. Obviously, this part (a) is similar to Fig. 1 of Ref. [16], which has shown the existence of a single free value of $\vartheta_{\mathcal{L}}^{+}(=0.0191 \pm 0.085)$ from a fit of the same data using the theoretical crossover max MR6 function [3] calculated at the time. In part (b) (and (c), see below Sect. 4.3), the results are reported over the experimental range illustrated by the horizontal (blue) segment labeled GC. We note that the corresponding calibration temperature of the Güttinger and Cannell's data is indicated by the vertical arrow labeled $\Delta \tau_{\mathrm{or}}^{*}$.

In the second case that accounts for the contribution of the leading term, it is necessary to introduce the following scaling relation between the effective amplitudes

$$
\Gamma_{e}^{+}=\left(\vartheta_{\mathcal{L}}^{+}\right)^{\gamma-\gamma_{e}} \mathbb{X}_{0, \mathcal{L}}^{*} \mathbb{Z}_{\chi, e}^{+}
$$

Now, the non-analytical scaling transformation

$$
f_{\gamma_{e}}\left(\mathbb{X}_{0, \mathcal{L}}^{*}, \vartheta_{\mathcal{L}}^{+}\right)=\mathbb{X}_{0, \mathcal{L}}^{*}\left(\vartheta_{\mathcal{L}}\right)^{\gamma-\gamma_{e}}
$$

is explicit in Eq. (27) and both constraints in position and direction are correctly taken into account. This transformation takes an effective power law form of the crossover parameter $\vartheta_{\mathcal{L}}^{+}$, while the prefactor $\mathbb{X}_{0, \mathcal{L}}^{*}$ has (as expected above) the same value whatever the value of $\gamma_{e, \text { expt }}\left(=\gamma_{e, \text { th }}\right)$. Equation (28) distinguishes each proper nature of the asymptotic prefactor $\mathbb{X}_{0, \mathcal{L}}^{*}$ and the field scale factor $\vartheta_{\mathcal{L}}^{+}$. Therefore, we can use the following equation

$$
\Gamma^{+}=\mathbb{X}_{0, \mathcal{L}}^{*}\left(\mathbb{Z}_{\chi}^{+}\right)^{-1}
$$




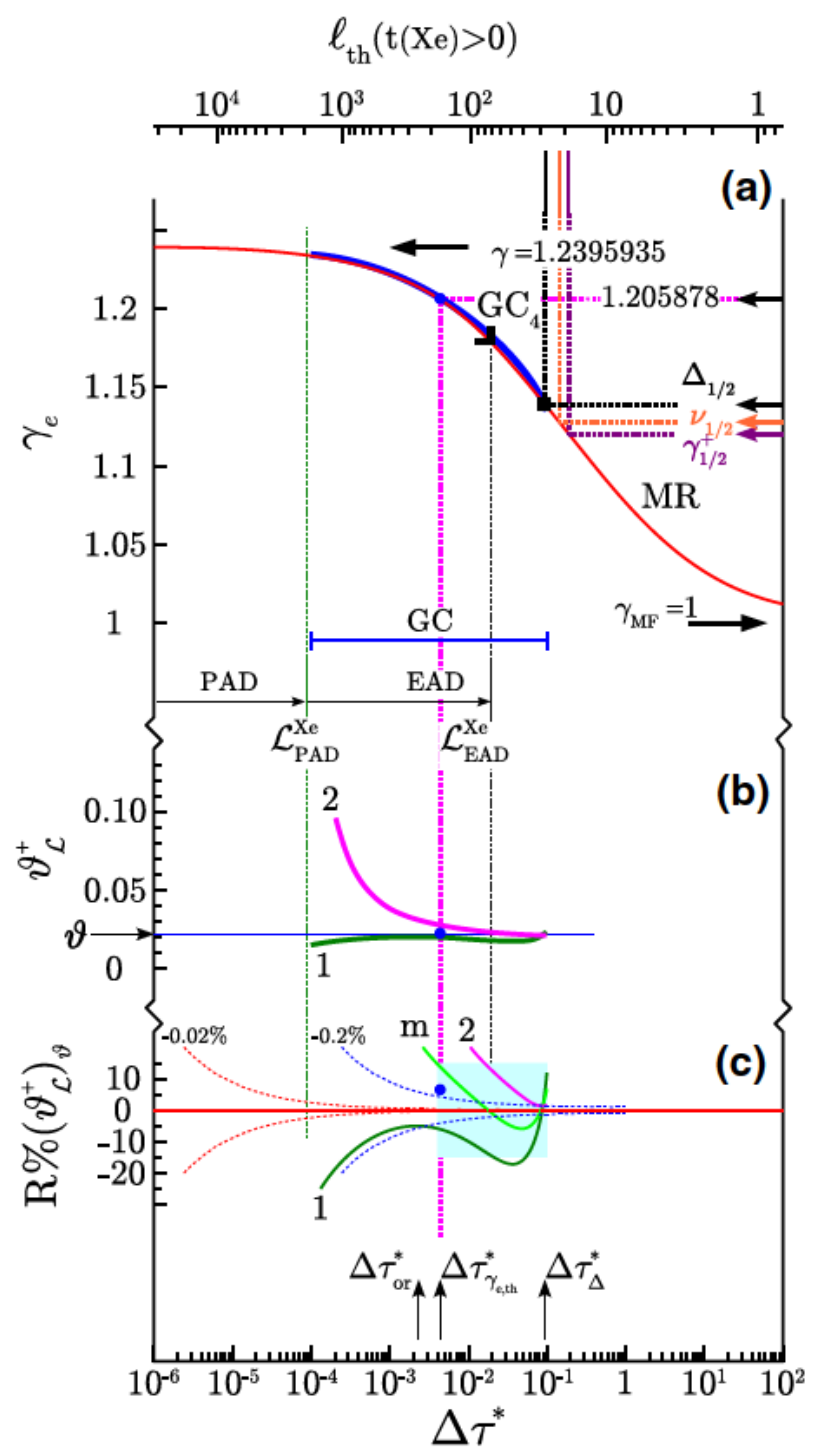

Fig. 2 Ising-like crossover behaviors of xenon susceptibility as a function of $\Delta \tau^{*}$ (lower horizontal axis) and $\ell_{\text {th }}$ in xenon case (upper horizontal axis). a $\gamma_{e}$-case. Red curve labeled MR: Eq. (18). Blue curve labeled GC : Eq. (20). Right corner and square: $\gamma_{e}$ at $\Delta \tau^{*}=\mathcal{L}_{\mathrm{EAD}}^{\mathrm{Xe}}$ and $\Delta \tau_{\Delta}^{*}=\frac{t_{\Delta}}{\vartheta}$, respectively, with $\vartheta=0.0211752$ (see text). $b \vartheta_{\mathcal{L}}^{+}$-case. (Green) curve 1: Eq. (26). (Pink) curve 2: Eq. (31). $c R \%(\vartheta)_{\vartheta}^{+}$-case: From $b$ with $R \%\left(\vartheta_{\mathcal{L}}^{+}\right)_{\vartheta}=100\left(\frac{\vartheta_{\mathcal{C}}^{+}}{\vartheta}-1\right)$. (Green light) curve m: mean value from Eqs. (26) and (31). Green area: $15 \%$ error-bar for the $\vartheta_{\mathcal{L}}^{+}=\vartheta$ matching. Symmetrical (blue and red) dotted curves: residual isoclines of Eq. (102) with $\delta \Gamma_{e}^{+}= \pm 0.2 \%$ and $\delta \Gamma_{e}^{+}= \pm 0.02 \%$ (see Appendix 2). Right side colored arrows: classical-to-critical crossing conditions defined in text. Blue points: corresponding results at $\Delta \tau_{\gamma_{e} \text {,th }}^{*}=4.347 \times 10^{-3}$ where $\gamma_{e}=1.205878$ (see Appendix 2). For other symbols and labels see text (Color figure online) 


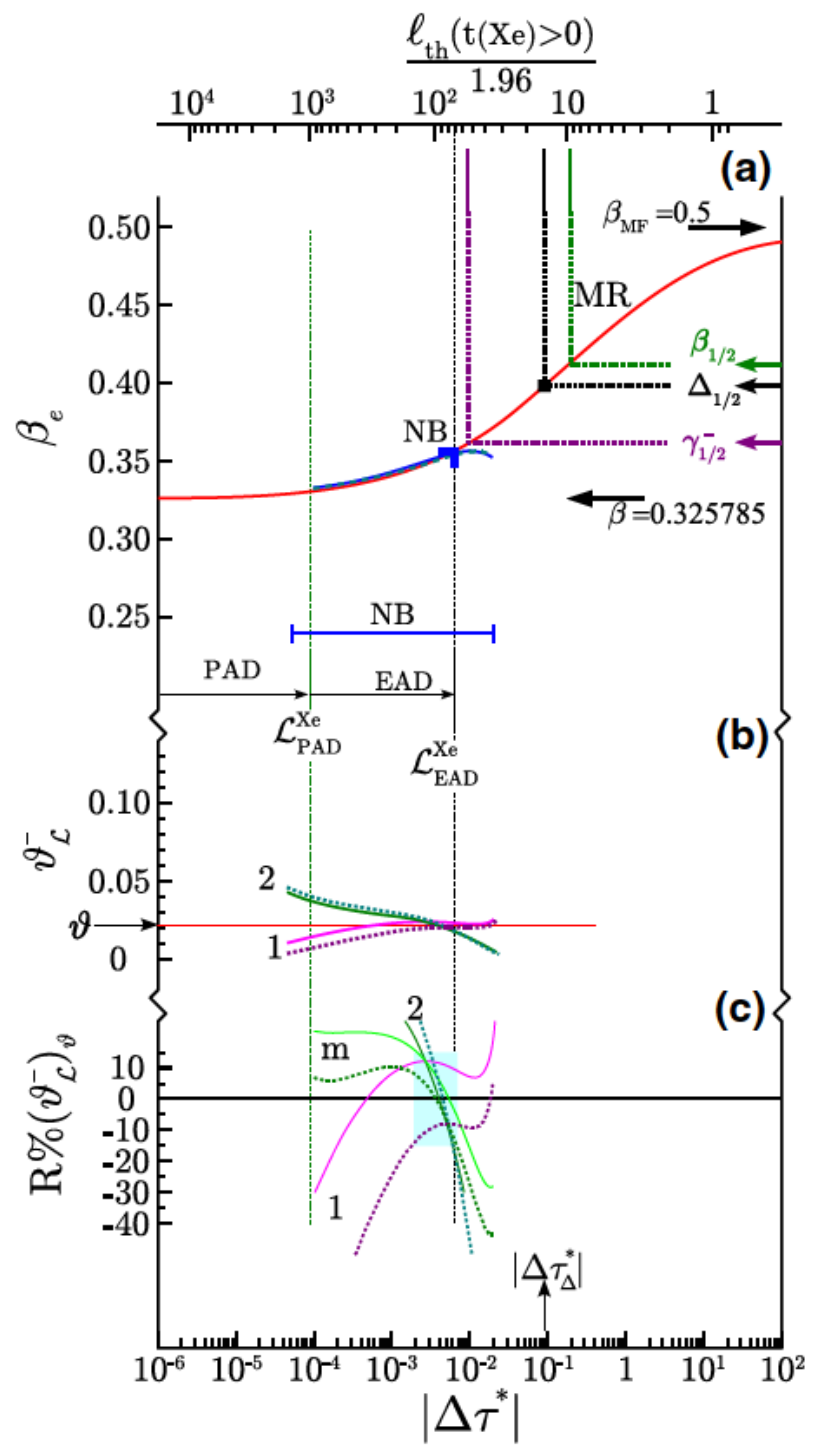

Fig. 3 Ising-like crossover behaviors of xenon order parameter density as a function of $\left|\Delta \tau^{*}\right|$ (lower horizontal axis) and $\ell_{\text {th }}^{-}=\frac{\ell_{\text {th }}}{1.96}$ (upper horizontal axis). a $\beta_{e}$-case. Red curve labeled MR: from $m_{\text {th }}(|t|)$ given in Ref. [9]. Blue curve labeled NB: from Eq. (34). Right corner and square $\beta_{e}$ at temperature distances $\left|\Delta \tau^{*}\right|=\mathcal{L}_{\mathrm{EAD}}^{\mathrm{Xe}}$ and $\left|\Delta \tau_{\Delta}^{*}\right|=\frac{t_{\Delta}}{\vartheta}$, respectively, with $\vartheta=0.0211752$ (see text). $b: \vartheta \overline{\mathcal{L}}^{- \text {case. (Pink full }}$ and dotted) curves 1: Eq. (38). (Green full and dotted) curves 2: Eq. (39). $c R \%(\vartheta \overline{\mathcal{L}})_{\vartheta}$-case: From $b$ with $R \%\left(\vartheta_{\mathcal{L}}\right)_{\vartheta}=100\left(\frac{\vartheta \overline{\mathcal{C}}}{\vartheta}-1\right)$. Curves m: mean values of Eqs. (26) and (39). Green area: $15 \%$ error-bar for the $\vartheta_{\mathcal{L}}=\vartheta$ matching. Right side colored arrows: different classical-to-critical crossing conditions defined in text. For other symbols and labels see text (Color figure online)

to eliminate $\mathbb{X}_{0, \mathcal{L}}^{*}$ and to introduce the true asymptotic amplitude (which fixes the position of the Ising-like limiting point of the experimental curve in Fig. 1). That infers the pure $\vartheta_{\mathcal{L}}$-dependence of the right-hand-side of equation 


$$
\frac{\Gamma_{e}^{+}}{\Gamma^{+}}=\left(\vartheta_{\mathcal{L}}^{+}\right)^{\gamma-\gamma_{e}} \frac{\mathbb{Z}_{\chi, e}^{+}}{\left(\mathbb{Z}_{\chi}^{+}\right)^{-1}}
$$

and leads to the unequivocal determination of $\vartheta_{\mathcal{L}}^{+}$when $\Gamma^{+}, \gamma_{e}$ and $\Gamma_{e}^{+}$are known, through the non-analytical scaling equation

$$
\vartheta_{\mathcal{L}}^{+}=\left(\frac{1}{\mathbb{Z}_{\chi}^{+} \mathbb{Z}_{\chi, e}^{+}} \times \frac{\Gamma_{e}^{+}}{\Gamma^{+}}\right)^{\frac{1}{\gamma-\gamma_{e}}}
$$

More generally, Eq. (31) valid for $\mathcal{L}_{\mathrm{PAD}}^{f}<\Delta \tau^{*} \lesssim \mathcal{L}_{\mathrm{EAD}}^{f}$, has equivalent Ising-like nature as the scaling equation $\vartheta=\left(\frac{a_{\chi}^{+}}{\mathbb{Z}_{\chi}^{1,+}}\right)^{\frac{1}{\Delta}}$ (expressing Eq. (6) for $\chi$ ), valid for $\Delta \tau^{*} \lesssim \mathcal{L}_{\mathrm{PAD}}^{f}$. In xenon case, we have numerically solved Eq. (31) at fixed $\gamma_{e}$, using Eq. (25) and $\chi_{\text {th }}(t)$ given in [9], with $\Gamma^{+}(\mathrm{Xe})=0.057824$. The resulting value $\vartheta_{\mathcal{L}}^{+}\left(\Delta \tau^{*}\right)$ corresponds to the curve labeled 2 in Fig. 2 b.

As previously mentioned, the upper horizontal axis gives the related value of $\ell_{t h}(t)$ with $t=\vartheta \Delta \tau^{*}>0$ and $\vartheta(\mathrm{Xe})=0.0211752$ (see Table 1 in Appendix 1). The values of $\gamma_{e}$ indicated by the black, red and brown horizontal arrows, respectively, correspond to the specific values $\Delta_{\frac{1}{2}}, v_{\frac{1}{2}}$, and $\gamma_{\frac{1}{2}}$. The corresponding values of $\ell_{t h}(t)$ are given by the respective colored vertical arrows in the range $\ell_{t h} \simeq 20-30$. The right corner symbol on the MR curve indicates the extension $\mathcal{L}_{\mathrm{EAD}}^{\mathrm{Xe}}$ of the Ising-like extended asymptotic domain (where $\ell_{t h} \simeq 2.6 \ell_{t h}\left(t_{\Delta}\right) \simeq 3 \mathbb{L}^{\{1 f\}} \simeq 75$ ). Assuming then that $\vartheta_{\mathcal{L}} \equiv \vartheta(\mathrm{Xe})=0.0211752$, the xenon extended asymptotic domain cannot exceed the following upper limit

$$
\Delta \tau^{*}<\mathcal{L}_{\mathrm{EAD}}^{\mathrm{Xe}}=\frac{\mathcal{L}_{\mathrm{EAD}}^{\mathrm{Ising}}}{\vartheta} \simeq 1.84 \times 10^{-2}
$$

i.e., $T-T_{c}<T_{c} \mathcal{L}_{\mathrm{EAD}}^{\mathrm{Xe}} \simeq 5.3 \mathrm{~K}$ in the homogeneous domain. Similarly, the square symbol corresponds to the master crossover temperature (where $\ell_{t h} \simeq \mathbb{L}^{\{1 f\}}=25.585$, i.e., when the correlation length is of the order of the size of the microscopic interaction). Two vertical dotted lines show the respective extensions $\mathcal{L}_{\mathrm{PAD}}^{\mathrm{Xe}}$ and $\mathcal{L}_{\mathrm{EAD}}^{\mathrm{Xe}}$ of the Ising-like pre- and extended asymptotic domains (see the horizontal arrows labeled PAD and EAD, respectively).

\subsection{The Order Parameter Density Case}

The above approach for the susceptibility case can be easily duplicated to the order parameter density case, introducing now the following arbitrary relation

$$
t=\vartheta_{\mathcal{L}}^{-}\left|\Delta \tau^{*}\right|
$$

We consider the vapor-liquid coexisting density data measured by Näger and Balzarini [34] in two different samples of xenon, which were fitted by the following (three term) Wegner-like expansion:

$$
\begin{gathered}
\Delta \tilde{\rho}_{L V, \operatorname{expt}}\left(\left|\Delta \tau^{*}\right|\right)=B\left|\Delta \tau^{*}\right|^{\beta}\left[1+a_{1 M}\left|\Delta \tau^{*}\right|^{\Delta}\right. \\
\left.+a_{2 M}\left|\Delta \tau^{*}\right|^{2 \Delta}\right]
\end{gathered}
$$

For the fits, the exponents $\beta=0.327$ and $\Delta=0.5$ were held fixed. For sample No. 1 , the corresponding values of the adjustable parameters are $B=1.479( \pm 0.011), a_{1 M}=$ $1.15( \pm 0.19), a_{2 M}=-2.6( \pm 1.0)$ (with an uncertainty of $\pm 1 \mathrm{mK}$ on the $T_{c}$ value). This fitting result is labeled $\mathrm{NB}_{3 a}$. For sample No. 2, the fit results are $B=1.470( \pm 0.010)$, 
$a_{1 M}=1.20( \pm 0.17), a_{2 M}=-2.8( \pm 1.1)$ (with an uncertainty of $\pm 2 \mathrm{mK}$ on the $T_{c}$ value), and the label is $\mathrm{NB}_{3 b}$.

We do not report here the similar complete descriptions of the effective power laws $\mathbb{Z}_{M, e}|t|^{\beta_{e}}$ and $B_{e}\left|\Delta \tau^{*}\right|^{\beta_{e}}$. We merely recall that they allow us to construct the theoretical and experimental curves of respective equations $\mathbb{Z}_{M, e}\left(\beta_{e}\right)$ and $B_{e}\left(\beta_{e}\right)$. The latter equations introduce the two-parameter transformation

$$
f_{\beta_{e}}\left(\mathbb{M}_{0, \mathcal{L}}^{*}, \vartheta_{\mathcal{L}}^{-}\right)=\mathbb{M}_{0, \mathcal{L}}^{*}\left(\vartheta_{\mathcal{L}}^{-}\right)^{\beta_{e}-\beta}
$$

which is similar to the one $f_{\gamma_{e}}\left(\mathbb{X}_{0, \mathcal{L}}^{*}, \vartheta_{\mathcal{L}}^{+}\right)=\mathbb{X}_{0, \mathcal{L}}^{*}\left(\vartheta_{\mathcal{L}}^{+}\right)^{\gamma-\gamma_{e}}$ for the susceptibility case [see Eq. (28)]. Thus the only needed material to obtain $\vartheta_{\mathcal{L}}^{-}$concerns the universal feature of the matching between the Ising-like limiting points at $\beta_{e}=\beta$. Here, the limiting transformation reads $f_{\beta}\left(\mathbb{M}_{0, \mathcal{L}}^{*}\right) \equiv \mathbb{M}_{0, \mathcal{L}}^{*}$. The corresponding fluid value of $\mathbb{M}_{0, \mathcal{L}}^{*}$ can be obtained using the following relations [11]

$$
\begin{aligned}
\mathbb{M}_{0, \mathcal{L}}^{*} & =\left(\mathbb{L}^{\{1 f\}}\right)^{d} \psi_{\rho} \vartheta^{\beta} \\
& =\left(\mathbb{L}^{\{1 f\}}\right)^{d} \Psi^{\{1 f\}}\left(\Theta^{\{1 f\}}\right)^{\beta}\left(Y_{c}\right)^{\beta}\left(Z_{c}\right)^{-\frac{1}{2}}
\end{aligned}
$$

which satisfy the two-scale factor universality through the analytical combination [see Eq. (16)] with $\mathbb{X}_{0, \mathcal{L}}^{*}$ of Eq. (24) and $\mathbb{L}_{0, \mathcal{L}}^{*}$ of following value [11]

$$
\begin{aligned}
\mathbb{L}_{0, \mathcal{L}}^{*} & =\left(\mathbb{L}^{\{1 f\}}\right)^{-1} \vartheta^{-v} \\
& =\left(\mathbb{L}^{\{1 f\}}\right)^{-1}\left(\Theta^{\{1 f\}}\right)^{-v}\left(Y_{c}\right)^{-v}
\end{aligned}
$$

The asymptotic dependence of the scale factors $\vartheta$ and $\psi_{\rho}$ is thus properly accounted for, i.e., in conformity with the two-scale factor universality of asymptotical scaling. In that pure power law scheme, the introduction of the true leading value of $B(\mathrm{Xe})=1.46762$ to replace $\mathbb{M}_{0, \mathcal{L}}^{*}$ complements our previous introduction of $\Gamma^{+}(\mathrm{Xe})=0.057824$ to replace $\mathbb{X}_{0, \mathcal{L}}^{*}$.

Accordingly, we can formulate two matching equations for $\beta_{e}$ and $\vartheta_{\mathcal{L}}^{-}$, similar to the ones for $\gamma_{e}$ [see Eq. (26)] and $\vartheta_{\mathcal{L}}^{+}$[see Eq. (31)]. In the first case using the single constraint in position, we numerically solve the following equation

$$
\beta_{e, \text { expt }}\left(\left|\Delta \tau^{*}\right|\right) \equiv \beta_{e, \text { th }}\left[\vartheta_{\mathcal{L}}^{-}\left(\left|\Delta \tau^{*}\right|\right)\right]
$$

The theoretical red curve labeled MR of equation $\beta_{e, \text { th }}\left[\vartheta\left(\left|\Delta \tau^{*}\right|\right)\right]$ and the experimental blue curve labeled NB of equation $\beta_{e \text { expt }}\left[\left(\left|\Delta \tau^{*}\right|\right)\right]$ are reported in Fig. 3(a). Note that the differences provided by the use of Eq. (34) with two different parameter sets that refer to the samples $\mathrm{N}^{\circ} 1$ and 2 , respectively, are not visible at the thickness scale of the experimental blue curve NB in this figure part (a). This part (a) is similar to Fig. 3 of Ref. [32], which was designed from the experimental data available at the time of the development of the min and max MR6 crossover functions, but not analyzed as the non-homogeneous domain was not theoretically investigated. The corresponding curves $\vartheta_{\mathcal{L}}^{-}\left(\left|\Delta \tau^{*}\right|\right)$ (with the label 1) are reported in Fig. $3 \mathrm{~b}$ (where $\mathrm{NB}_{3 a}$ corresponds to the full pink line, while $\mathrm{NB}_{3 b}$ corresponds to the dotted pink line).

In the second case, using both constraints in direction and position, the pure $\vartheta_{\mathcal{L}^{-}}^{-}$ dependence is obtained computing numerically the non-analytical scaling equation

$$
\vartheta_{\mathcal{L}}^{-}=\left(\frac{\mathbb{Z}_{M}}{\mathbb{Z}_{M, e}} \times \frac{B_{e}}{B}\right)^{\frac{1}{\beta_{e}-\beta}}
$$


Equation (39) is similar with Eq. (31) and thus Ising-like similar with Eq. (6) expressed for the order parameter case $\left(\vartheta=\left(\frac{a_{M}}{\mathbb{Z}_{M}^{1}}\right)^{\frac{1}{\Delta}}\right)$. The curves $\vartheta_{\mathcal{L}}^{-}\left(\left|\Delta \tau^{*}\right|\right)$ of Eq. (39) are labeled 2 in Fig. $3 b$ (with a full green line for $\mathrm{NB}_{3 a}$ and a dotted green line for $\mathrm{NB}_{3 b}$ ).

In Fig. 3, the available (experimental) temperature range is now illustrated by the horizontal blue segment labeled NB and all the other symbols and labels are similar to the ones in Fig. 2 for the isothermal compressibility case. As the theoretical crossover function of the correlation length in the non homogeneous domain is not given in Ref. [5], we have used the realistic approximation $\ell_{t h}^{-}(t<0)=\frac{\ell_{t h}(t>0)}{1.96}$ to label the upper horizontal axis of Fig. 3 . The values of $\beta_{e}$ indicated by the black, green and purple horizontal arrows, respectively, correspond to $\Delta_{\frac{1}{2}}, \beta_{\frac{1}{2}}$, and $\gamma_{\frac{1}{2}}^{-}$(in the non homogeneous region). The related values of $\ell_{t h}^{-}(t)$ are given by the respective colored vertical arrows in the range $\ell_{t h}^{-} \simeq 10-60$. Here we observe that the crossing temperature for $\gamma_{\frac{1}{2}}^{-}$is slightly greater than $\mathcal{L}_{\mathrm{EAD}}^{\mathrm{Xe}}$ (where $\ell_{\text {th }}^{-} \simeq 2.6 \ell_{\text {th }}^{-}\left(t_{\Delta}\right) \simeq 3 \mathbb{L}^{\{1 f\}} \simeq 75$ ) but lower than $\left|\Delta \tau_{\Delta}^{*}\right|=\frac{t_{\Delta}}{\vartheta} \simeq 9 \times 10^{-2}$ (see the lower vertical arrow in Fig. 3c), where $\ell_{t h}^{-} \simeq \mathbb{L}^{\{1 f\}}=25.585$.

\subsection{Comparison Between $\vartheta \stackrel{\mathcal{L}}{ \pm}$ and $\vartheta$}

The relative comparison between $\vartheta_{\mathcal{L}}^{ \pm}$and $\vartheta$ values for xenon is made in parts (c) of Figs. 2 and 3, using the residuals $R \%\left(\vartheta_{\mathcal{L}}^{ \pm}\right)_{\vartheta}=100\left(\frac{\vartheta_{\mathcal{L}}^{ \pm}}{\vartheta}-1\right)$ (expressed in $\%$ ). In each figure the residuals refer to $\vartheta_{\mathcal{L}}^{ \pm}$obtained as a local numerical solution of Eqs. (26) and (38) (curves labeled 1) and Eqs. (31) and (39) (curves labeled 2) respectively. The curves labeled $m$ correspond to the respective mean values between curves 1 and 2 . In the range $\Delta \tau^{*}<$ $10^{-2}$, a noticeable increase of the residuals is observed approaching the critical temperature. That reflects the difference between the Ising values of the critical exponent $(\gamma=1.241$, $\beta=0.327)$ used in the experimental fits and the ones $(\gamma=1.2395935, \beta=0.3257845)$ used in the MR scheme. The upper limit of the fitting agreement with a single crossover parameter corresponds to the abrupt increase of the residuals at large temperature range (i.e., $\Delta \tau^{*}>10^{-1}$ in Fig. $2 \mathrm{c}$ and $\left|\Delta \tau^{*}\right|>2 \times 10^{-2}$ in Fig. $3 \mathrm{c}$ ). The expected identity $\vartheta \equiv \vartheta_{\mathcal{L}}^{ \pm}$in xenon case, is here observed in the green area on each figure. The error-bar of $\pm 15 \%$ underlined in this green area corresponds to an error-bar lower than $1 \%$ for the related effective amplitude (see Appendix 2 in the $\Gamma_{e}^{+}$case). The corresponding values $1.5 \times 10^{-2} \lesssim \Delta \tau^{*} \lesssim 10^{-1}$ in Fig. $2 \mathrm{c}$ and $2 \times 10^{-3} \lesssim\left|\Delta \tau^{*}\right| \lesssim 7 \times 10^{-3}$ in Fig. $3 \mathrm{c}$, are at least one and a half order of magnitude larger than $\mathcal{L}_{\mathrm{PAD}}^{\mathrm{Xe}} \simeq 0.9 \times 10^{-4}$. Moreover, as clearly indicated on both figures, the condition $\mathcal{L}_{\mathrm{EAD}}^{\mathrm{Xe}}<\Delta \tau_{\Delta}^{*}$ demonstrates the Ising-like nature of the extended asymptotic domain for critical xenon and confirms the scaling role of Eqs. (31) and (39).

Before to formulate the conclusive remarks, it is essential to recall the main consequences of the identity $\vartheta \equiv \vartheta_{\mathcal{L}}^{ \pm}$. This identity was validated from two distinct properties measured at finite distance from $T_{c}$ along the critical isochore, above and below $T_{c}$. The three-parameters characterization of the asymptotic Ising-like nature of xenon was then given by the amplitude set $\left\{a_{\chi}^{+} ; \xi^{+} ; \Gamma^{+}\right\}$of Table 1 in Appendix 1 , or the MR parameter set $\left\{\vartheta ; g_{0} ; \psi_{\rho}\right\}$ of same Table 1, while the Ising-like universal features were validated by the control of the PAD description for the selected three different experimental properties. Therefore, $\vartheta$ is well the single non-universal scale factor that characterizes the xenon critical isochore, while, as a 
correlated Ising-like result, $\psi_{\rho}$ is well the single non-universal scale factor that characterizes the xenon critical isotherm (despite a non available validation from the analysis of a still missing measurement of a xenon singular property along the critial isotherm). These two, non-dimensional, characteristic scale factors $\vartheta$ and $\psi_{\rho}$ introduced in the MR theoretical asymptotic description of the $O(1)$ universality class are linked, respectively, to the two, non-dimensional characteristic critical factors $Y_{c}$ and $Z_{c}$ defined at the exact critical point from the master phenomenological description of the one-component fluid subclass. The links only need to use the unique length unit for correlation and thermodynamic dimensional properties, where it is obvious that the master quantity $\mathbb{L}^{\{1 f\}}$ has thus the subtle double role to get simultaneously the required link between $\alpha_{c}$ and $\left(g_{0}\right)^{-1}$ and to account for critical length dimensions of the bare (physical) quantities [9]. Finally, the above xenon description validated at finite distance from $T_{c}$ includes the implicit interpolated PAD description. Here we recall that interpolated means that the resulting theoretical description of the temperature range $0<\Delta \tau^{*} \lesssim \mathcal{L}_{\mathrm{PAD}}^{\mathrm{Xe}}$ occurs without the support of the experimental data, only using the relative susceptibility data and the relative turbidity data of Güttinger and Cannell [20] (added to their corresponding reference susceptibility and turbidity data at $T_{c}+0.6677 \mathrm{~K}$ ), and the vapor-liquid coexisting density data of Näger and Balzarini [34], measured in the temperature range $\mathcal{L}_{\mathrm{PAD}}^{\mathrm{Xe}}<\left|\Delta \tau^{*}\right| \leq \mathcal{L}_{\mathrm{EAD}}^{\mathrm{Xe}}$.

\section{Remarks and Conclusion}

As the single numerical value of the non-universal parameters $\vartheta_{\mathcal{L}}^{ \pm}$appears to be governed by the susceptibility and coexisting density data measurements at the largest possible temperature distance beyond the Ising-like preasymptotic domain, we can formulate two summarizing remarks before concluding.

\subsection{Similarity Between the Three-Parameter Crossover Models}

The first remark concerns the impact of the application of different crossover models on the fitting results obtained using the same data measurements. Indeed, for all the models, it appears that a single free parameter can be defined as a non-universal temperature scaling factor (so-called temperaturelike Ginzburg number), similar to $\left(\vartheta_{\mathcal{L}}^{ \pm}\right)^{-1}$. Such a result can be explained only assuming a remarkable collapse of their theoretical Ising-like crossover shapes on the upgraded MR7 ones, only using the theoretical estimates of three universal independent amplitudes involved in each model PAD description. This collapse is expected similar to the MR-master collapse described in Ref. [11] and recalled in Appendix 1. That expected modelling similarity will be formalized in a forthcoming paper.

\subsection{Effective Singular Behavior of the One Component Fluids at Finite Temperature Distance from $T_{c}$}

The second remark, complemented by the detailed analysis of the Güttinger and Cannell's susceptibility data given in Appendix 2, provides an additional view of the effective universality analyzed in the 80's on the basis of scaled equations of state formulated in terms of two non-universal parameters. The finite temperature range such as $\Delta \tau^{*}>\mathcal{L}_{\mathrm{PAD}}^{f}$ was largely investigated in the seventies [41], when the scaling approach of fluid universality was based on the effective "universal" values of the exponents (as for example $\gamma_{e \text {,EOS }}=1.19$, and $\beta_{e, \mathrm{EOS}}=0.355[41,42]$ involved in the effective rescaled formulation of a parametric 
equation of state). Today, it is well-admitted that the effective critical exponents introduce an inconsistency into the use of renormalization group results which clouds the analysis of experimental data. However, in that effective description, the Ising-like nature of the fluid $f$ was mainly accounted for by introducing only two adjustable parameters in the equation of state, as only two effective exponents are assumed free (an uncorrect hypothesis since only two critical exponents are free in the critical asymptotic limit $\Delta \tau^{*} \rightarrow 0$ ). Despite this theoretical inconsistency, the practical number of fluid-dependent parameters was then conform to our present analysis in the intermediate temperature range $\mathcal{L}_{\mathrm{PAD}}^{f} \leq \Delta \tau^{*} \leq \Delta \tau_{\Delta}^{*}$. In the light of the present work, the main reason which explains this effective scaled universality [43] is not directly the two-scale factor universality but the uniqueness of the scale factor which characterizes the confluent corrections to scaling due to a single irrelevant scaling field, as demonstrated precisely in Appendix 2.

\subsection{Conclusion}

This work, restricted to the simple fluid critical isochore to control the number (three) of fluid-dependent parameters, gives a new and well-defined procedure to analyze the experimental results selecting one particular theoretical method (field theory crossover) to obtain accurate results along this critical path. Indeed the three fluid-dependent parameters $\vartheta, g_{0}$, and $\psi_{\rho}$ needed to take benefit of the Ising-like universal features predicted by the massive renormalization scheme asymptotically close to the non-trivial fixed point can be predicted from the four generalized coordinates of the vapor-liquid critical point. Using such expected description only valid within the Ising-like preasymptotic domain, we have shown that, when the energy $\left(k_{B} T_{c}\right)$ and length $\left(\alpha_{c}\right)$ units are known, the mean crossover functions for both the susceptibility in the homogeneous domain and the order parameter density in the non homogeneous domain provide access to the convenient explicit scaling equations (above and below $T_{c}$ ) to determine the local value of a single non-universal parameter $\vartheta_{\mathcal{L}}^{ \pm}$in both ( $T \gtrless T_{c}$ ) temperature ranges beyond the Ising-like preasymptotic domain. The Ising-like, scale-factor nature of this critical parameter remains evident using the dimensionless master value $\mathbb{L}^{\{1 f\}}=\alpha_{c} g_{0}=25.585$ of a single characteristic length of the one-component fluid subclass. We have then clearly shown that the resulting value of the non-analytic scaling equations are entirely governed by the data measurements which are accounted for correctly by the theoretical crossover description at the largest possible distance from the critical point. Finally, the magnitude of the resulting deviations and the temperature range where these deviations become significant to invalidate the uniqueness of the non-universal parameter defined by the identity $\vartheta \equiv \vartheta_{\mathcal{L}}^{ \pm}$, are accounted for exactly in the Ising-like extended asymptotic domain $\Delta \tau^{*} \leq \mathcal{L}_{\mathrm{EAD}}^{f}$, corresponding to the theoretical correlation length range $\ell_{t h}\left(t=\vartheta \Delta \tau^{*}\right) \gtrsim 2.6 \ell_{t h}\left(t_{\Delta}=\vartheta \Delta \tau_{\Delta}^{*}\right) \simeq 3 \mathbb{L}^{\{1 f\}} \simeq 75$. Here $t_{\Delta}=\left(\frac{1}{S_{2}}\right)^{2} \cong 1.9 \times 10^{-3}$ is the crossover temperature that characterizes the exchange between the prominent Ising-like nature of the confluent exponent due to the value $\Delta=0.50189$ approaching the non-trivial fixed point $\left[t \ll t_{\Delta}\right]$, and its prominent mean-field-like nature due to the value $\Delta_{\mathrm{MF}}=\frac{1}{2}$ approaching the Gaussian fixed point $\left[t \gg t_{\Delta}\right]$. The main consequence is that the relevant scaling field and the single irrelevant confluent field have the same Ising-like, fluid-dependent scale factor along the critical isochore. Xenon is selected here as being the single onecomponent fluid with accurate measurements of two distinct singular properties above and below $T_{c}$ to support the procedure. Therefore, thanks to the precise estimates of the universal features from the MR scheme along the primary path at $h=0$, xenon close to its vapor-liquid critical point is now an Ising-like standard without adjustable parameters along its critical 
isochore, similar to the $O(1)$ symmetric $\left(\Phi^{2}\right)^{2}$ field theory and the $N=1$-vector model of three-dimensional (3D) Ising-like systems.

From this Ising-like standard situation provided by critical xenon, the real extension and amplitude of the singular behavior of the fluid properties can be estimated in a similar manner for any one-component fluid for which the vapor-liquid critical point is localized in the $p V T$ phase surface, thanks to the use of the master crossover functions given in [11] and/or the CMM model of the equation of state given in [21]. Therefore, the comparisons between simple fluid experiments and theory will be improved as they can be performed, now [44] and in the future, without adjustable non-universal parameter.

Acknowledgments We acknowledge F. Palencia for his contribution to the development of Mathematica files. Part of the work was supported by CNES.

\section{Appendix 1: Expected Fluid Characterization Within the PAD}

\section{MR Characterization of the PAD for the $O$ (1) Universality Class}

The PAD description from the three theoretical mean crossover functions [9] of present interest writes as follows:

$$
\begin{aligned}
\ell_{\mathrm{PAD}, \text { th }}(t) & =\left(\mathbb{Z}_{\xi}^{+}\right)^{-1} t^{-v}\left(1+\mathbb{Z}_{\xi}^{1,+} t^{\Delta}\right) \\
\chi_{\mathrm{PAD}, \text { th }}(t) & =\left(\mathbb{Z}_{\chi}^{+}\right)^{-1} t^{-\gamma}\left(1+\mathbb{Z}_{\chi}^{1,+} t^{\Delta}\right) \\
m_{\mathrm{PAD}, \text { th }}(|t|) & =\mathbb{Z}_{M}|t|^{\beta}\left(1+\mathbb{Z}_{M}^{1}|t|^{\Delta}\right)
\end{aligned}
$$

where the theoretical amplitude set,

$$
\mathbb{S}_{A}^{\{M R\}}=\left\{\begin{array}{c}
\mathbb{Z}_{\chi}^{1,+}=8.56347 \\
\left(\mathbb{Z}_{\xi}^{+}\right)^{-1}=0.471474 \\
\left(\mathbb{Z}_{\chi}^{+}\right)^{-1}=0.269571
\end{array}\right\}
$$

is here selected to characterize the universal asymptotic singular behavior of the $N=1$-vector model in three dimension for the $O(1)$ universality class. ${ }^{2}$ Indeed, in Eqs. (40) to (42), the remaining theoretical amplitudes $\mathbb{Z}_{\xi}^{1,+}=5.81623, \mathbb{Z}_{M}=0.937528$, and $\mathbb{Z}_{M}^{1}=7.70712$ are conform with the following universal combinations

$$
\begin{aligned}
\left(\mathbb{Z}_{\xi}^{+}\right)^{-d} \mathbb{Z}_{\chi}^{+}\left(\mathbb{Z}_{M}\right)^{2} & =\frac{\left(R_{\xi}^{+}\right)^{d}}{R_{C}}=Q_{c} \\
\frac{\mathbb{Z}_{\xi}^{1,+}}{\mathbb{Z}_{\chi}^{1,+}} & =0.67919 \\
\frac{\mathbb{Z}_{M}^{1}}{\mathbb{Z}_{\chi}^{1,+}} & =0.9
\end{aligned}
$$

2 The related exponent set is $\{v=0.6303875, \gamma=1.2395935, \Delta=0.50189\}$, with the quoted precision needed by the contruction of the mean crossover functions [9] (as mentioned in the Introduction). Consequently, $\beta=\frac{1}{2}(d v-\gamma)=0.3257845$ in Eq. (42) and $\alpha=2-d v=0.1088375$ when the specific heat case is added to close the hyperscaling universality analysis along the critical isochore. 
with $R_{C}=\frac{\alpha \mathbb{Z}_{C}^{+}}{\mathbb{Z}_{\chi}^{+}\left(\mathbb{Z}_{M}\right)^{2}}=0.0574, R_{\xi}^{+}=\left(\mathbb{Z}_{\xi}^{+}\right)^{-1}\left(\alpha \mathbb{Z}_{C}^{+}\right)^{\frac{1}{d}}=0.2697$, and $Q_{c}=0.3418$ [8] (see the definition of $Q_{c}$ in the Introduction). The theoretical amplitude $\mathbb{Z}_{C}^{+}=1.719788$ corresponds to the specific heat case above $T_{c}{ }^{3}$

The estimated validity range $t \leq \mathcal{L}_{\mathrm{PAD}}^{\text {Ising }}$ of Eqs. (44) to (46) extends to

$$
\mathcal{L}_{\mathrm{PAD}}^{\mathrm{Ising}} \simeq \varpi\left(\frac{1}{S_{2}}\right)^{2} \cong 1.9 \times 10^{-6}
$$

with $\varpi=10^{-3}$ and $S_{2}=22.9007$ [9].

\section{Master Description of the PAD for the $\{1 f\}$-Universality Subclass}

The above MR PAD description can now be restricted to the so-called master description of the PAD for the $\{1 f\}$-subclass [11], by introducing only three constant scale factors $\Theta^{\{1 f\}}$, $\Psi^{\{1 f\}}, \mathbb{L}^{\{1 f\}}$, through the following asymptotic equations:

$$
\begin{array}{ccc}
t & =\Theta^{\{1 f\}} \mathcal{T}^{*} \\
h & =\Psi^{\{1 f\}} \mathcal{H}^{*} \\
\ell_{\text {PAD,th }}(t) & =\mathbb{L}^{\{1 f\}} \ell^{*}\left(\mathcal{T}^{*}\right)
\end{array}
$$

where $\mathcal{T}^{*}$ and $\mathcal{H}^{*}$ are the master temperature-like and magnetic-like fields, respectively. The master constant set $S_{S C}^{\{1 f\}}$,

$$
\mathcal{S}_{S C}^{\{1 f\}}=\left\{\begin{array}{l}
\Theta^{\{1 f\}}=4.288 \times 10^{-3} \\
\mathbb{L}^{\{1 f\}}=25.585 \\
\Psi^{\{1 f\}}=1.75505 \times 10^{-4}
\end{array}\right\}
$$

characterizes the $\{1 f\}$-subclass to be conform with the universality class of the threedimensional, uni-axial, Ising-like systems. Indeed, the three corresponding master equations can be written as follows:

$$
\begin{aligned}
\ell^{*}\left(\mathcal{T}^{*}\right) & =\mathcal{Z}_{\xi}^{+} \mathcal{T}^{*-v}\left(1+\mathcal{Z}_{\xi}^{1,+} \mathcal{T}^{* \Delta}\right)=\left(\mathbb{L}^{\{1 f\}}\right)^{-1} \ell_{\mathrm{PAD}, \mathrm{th}}(t) \\
\mathcal{X}^{*}\left(\mathcal{T}^{*}\right) & =\mathcal{Z}_{\chi}^{+} \mathcal{T}^{*-\gamma}\left(1+\mathcal{Z}_{\chi}^{1,+} \mathcal{T}^{* \Delta}\right)=\left(\mathbb{L}^{\{1 f\}}\right)^{d}\left(\Psi^{\{1 f\}}\right)^{2} \chi_{\mathrm{PAD}, \mathrm{th}}(t) \\
\mathcal{M}\left(\left|\mathcal{T}^{*}\right|\right) & =\mathcal{Z}_{M}\left|\mathcal{T}^{*}\right|^{\beta}\left(1+\mathcal{Z}_{M}^{1}\left|\mathcal{T}^{*}\right|^{\Delta}\right)=\left(\mathbb{L}^{\{1 f\}}\right)^{d} \Psi^{\{1 f\}} m_{\mathrm{PAD}, \mathrm{th}}(|t|)
\end{aligned}
$$

where the master amplitude set,

$$
\mathcal{S}_{A}^{\{1 f\}}=\left\{\begin{array}{ccc}
\mathcal{Z}_{\chi}^{1,+}= & \mathbb{Z}_{\chi}^{1,+}\left(\Theta^{\{1 f\}}\right)^{\Delta} & =0.555 \\
\mathcal{Z}_{\xi}^{+}= & \left(\mathbb{L}^{\{1 f\}}\right)^{-1}\left(\Theta^{\{1 f\}}\right)^{-\nu}\left(\mathbb{Z}_{\xi}^{+}\right)^{-1} & =0.5729 \\
\mathcal{Z}_{\chi}^{+}= & \left(\mathbb{L}^{\{1 f\}}\right)^{d}\left(\Psi^{\{1 f\}}\right)^{2}\left(\Theta^{\{1 f\}}\right)^{-\gamma}\left(\mathbb{Z}_{\chi}^{+}\right)^{-1}=0.11975
\end{array}\right\}
$$

then characterizes the master descriptions of the PAD for the $\{1 f\}$-subclass. Additional master amplitudes $\mathcal{Z}_{\xi}^{1,+}=\mathbb{Z}_{\xi}^{1,+}\left(\Theta^{\{1 f\}}\right)^{\Delta}=0.37695, \mathcal{Z}_{M}=\left(\mathbb{L}^{\{1 f\}}\right)^{d} \Psi^{\{1 f\}}\left(\Theta^{\{1 f\}}\right)^{\beta} \mathbb{Z}_{M}=$ 0.4665 , and $\mathcal{Z}_{M}^{1}=\mathbb{Z}_{M}^{1}\left(\Theta^{\{1 f\}}\right)^{\Delta}=0.4995$ are conform with Eqs. (44) to (46). For the

\footnotetext{
${ }^{3}$ Our present scaling approach uses the correlation length and the susceptibility as independent properties in terms of the two-scale-factor universality. We can then ignore the universal critical value of the background constant of the heat capacity, which is generally mixed with the non-universal contribution of the regular background terms in the one-component fluid case.
} 
specific heat case above $T_{c}$, the master amplitude $\mathcal{Z}_{C}^{+}=\left(\mathbb{L}^{\{1 f\}}\right)^{d}\left(\Theta^{\{1 f\}}\right)^{2-\alpha} \alpha \mathbb{Z}_{C}^{+}=$ 0.104324 closes the hyperscaling universality in conformity with the universal value of $R_{\xi}^{+}=\left(\mathcal{Z}_{C}^{+}\right)^{\frac{1}{d}} \mathcal{Z}_{\xi}^{+}=0.2696$ (with $R_{C}=\frac{\mathcal{Z}_{C}^{+} \mathcal{Z}_{\chi}^{+}}{\left(\mathcal{Z}_{M}\right)^{2}}$ ).

The master extension $\mathcal{L}_{\mathrm{PAD}}^{\{1 f\}}$ of the Ising-like preasymptotic domain of the $\{1 f\}$-subclass corresponds to

$$
\mathcal{L}_{\mathrm{PAD}}^{\{1 f\}}=\frac{\mathcal{L}_{\mathrm{PAD}}^{\text {Ising }}}{\Theta^{\{1 f\}}} \cong 4.7 \times 10^{-4}
$$

\section{Physical Description of the PAD for a (Non-quantum) One-Component Fluid}

\section{Three Amplitude Characterization of the Simple Fluid PAD}

Dimensionless Eq. (1) for the correlation length $\xi_{\text {expt }}^{*}\left(\Delta \tau^{*}\right)$ and the isothermal compressibility $\kappa_{T \text {,expt }}^{*}\left(\Delta \tau^{*}\right)$ in the homogeneous domain $\Delta \tau^{*}>0$, and for the spontaneous non-zero value of the (symmetrical) order parameter density $\Delta \tilde{\rho}_{L V \text {,expt }}\left(\left|\Delta \tau^{*}\right|\right)$ in the nonhomogeneous domain $\Delta \tau^{*}<0$, can be written as follows

$$
\begin{aligned}
\xi_{\text {expt }}^{*}\left(\Delta \tau^{*}\right) & =\xi^{+}\left(\Delta \tau^{*}\right)^{-v}\left[1+a_{\xi}^{+}\left(\Delta \tau^{*}\right)^{\Delta}\right] \\
\kappa_{T, \text {expt}}^{*}\left(\Delta \tau^{*}\right) & =\Gamma^{+}\left(\Delta \tau^{*}\right)^{-\gamma}\left[1+a_{\chi}^{+}\left(\Delta \tau^{*}\right)^{\Delta}\right] \\
\Delta \tilde{\rho}_{L V, \text { expt }}\left(\left|\Delta \tau^{*}\right|\right) & =B\left|\Delta \tau^{*}\right|^{\beta}\left[1+a_{M}\left|\Delta \tau^{*}\right|^{\Delta}\right]
\end{aligned}
$$

where

$$
\Delta \tilde{\rho}_{L V, \operatorname{expt}}=\frac{\rho_{L}-\rho_{V}}{2 \rho_{c}}
$$

is the reduced density difference between the liquid $\left(\rho_{L}\right)$ and vapor $\left(\rho_{V}\right)$ coexisting phases.

Among the three leading amplitudes $\xi^{+}, \Gamma^{+}$and $B$, only two are characteristic of the fluid while among the three confluent amplitudes $a_{\xi}^{+}, a_{\chi}^{+}$, and $a_{M}$, only one is a characteristic crossover-like parameter of the fluid. Selecting here the characteristic amplitude set

$$
S_{A, f}=\left\{a_{\chi}^{+} ; \xi^{+} ; \Gamma^{+}\right\}
$$

the remaining amplitudes $B, a_{\xi}^{+}$, and $a_{M}$ of Eqs. (55) to (57) can thus be calculated using the universal amplitude ratios and combinations of Eqs. (44) to (46) with $R_{\xi}^{+}=\left(A^{+}\right)^{\frac{1}{d}} \xi^{+}$and $R_{C}=A^{+} \frac{\Gamma^{+}}{B^{2}} . A^{+}$is the additional leading amplitude of the asymptotic singular behavior of the specific heat at constant volume along the critical isochore in the homogeneous domain $\Delta \tau^{*}>0$.

\section{Four Critical Parameters as Entry Data}

The entry material to estimate $S_{A, \mathrm{Xe}}=\left\{a_{\chi}^{+} ; \xi^{+} ; \Gamma^{+}\right\}$is provided by the following minimal set of four critical coordinates (neglecting here the quantum effects $[39,45]$ )

$$
Q_{c, a_{\bar{p}}}^{\min }=\left\{T_{c} ; p_{c} ; v_{\bar{p}, c} ; \gamma_{c}^{\prime}\right\}
$$

which localizes the fluid critical point on the phase surface of equation of state $\Phi_{a_{\bar{p}}}^{p}\left(p, v_{\bar{p}}, T\right)$ $=0$. The subscript $a_{\bar{p}}$ recalls for a thermodynamic description that starts from the Helmholtz 
free energy of the xenon particle. $v_{\bar{p}}=\frac{m_{\bar{p}}}{\rho}=\frac{1}{n}$ is the molecular volume and $\gamma_{c}^{\prime}$ is the common critical direction at the critical temperature of the critical isochoric line and the saturation pressure curve in the $p ; T$ diagram.

Using Eq. (60), the length and energy units are

$$
\begin{aligned}
\alpha_{c} & =\left(\frac{k_{B} T_{c}}{p_{c}}\right)^{\frac{1}{d}}=\left(\beta_{c} p_{c}\right)^{-\frac{1}{d}} \\
\left(\beta_{c}\right)^{-1} & =k_{B} T_{c}
\end{aligned}
$$

respectively (with $d=3$ ), while $p_{c} \sim\left[\frac{\text { energy }}{\text { volume }}\right]$ scales all the energy densities for a fluid of constant total volume unit $(V=1)$. Therefore, $\xi_{\text {expt }}^{*}=\frac{\xi_{\text {expt }}}{\alpha_{c}}$ in Eq. (55) and $\kappa_{T \text {,expt }}^{*}=$ $\kappa_{T, \operatorname{expt}} p_{c}$ in Eq. (56), with $\xi^{+}=\frac{\xi_{0}^{+}}{\alpha_{c}}$ and $\Gamma^{+}=\Gamma_{0}^{+} p_{c}$, where $\xi_{\text {expt }}, \kappa_{T, \text { expt }}, \xi_{0}^{+}$, and $\Gamma_{0}^{+}$ are the related dimensional quantities. The superscript star labels dimensionless quantities obtained only using $\alpha_{c}$ and $\left(\beta_{c}\right)^{-1}$ when the thermodynamic properties are normalized per particle [28-30].

However, Eq. (58) also introduces the practical dimensionless form $\tilde{\rho}=\frac{\rho}{\rho_{c}}$ of the (mass) density, which complements the practical dimensionless forms $\tilde{\mu}=\frac{\mu_{\rho} \rho_{c}}{p_{c}}$ of the conjugated chemical potential $\mu_{\rho}$ per mass unit $[37,42]$. The practical dimensionless variables that use critical parameters such as $\rho_{c}$ and $\frac{\rho_{c}}{p_{c}}$ are then decorated by a tilde. The definition of $\tilde{\mu}_{\rho}$ introduces another unit $\frac{p_{c}}{\rho_{c}} \sim\left[\frac{\text { energy }}{\text { mass }}\right]$ for specific energies, which differs from $\left(m_{\bar{p}} \beta_{c}\right)^{-1}$ by the usual critical compressibility factor $Z_{c}$ defined as

$$
Z_{c}=\frac{\frac{p_{c}}{\rho_{c}}}{\left(m_{\bar{p}} \beta_{c}\right)^{-1}}=\frac{p_{c} m_{\bar{p}}}{\rho_{c} k_{B} T_{c}}
$$

$m_{\bar{p}}=\frac{M}{N}$, where $M$ and $N$ are the total mass and total particle number of the fluid system, respectively. Similarly, the constant total mass unit $(M=1)$ of the fluid system introduces the critical specific volume $v_{c, M=1}=\frac{1}{\rho_{c}}$, which differs from the volume of the critical interaction cell $v_{c, I}=\frac{k_{B} T_{c}}{p_{c}}=\left(\alpha_{c}\right)^{d}$ [28] by the critical mass factor $\frac{m_{\bar{p}}}{Z_{c}}=\frac{v_{c, M=1}}{v_{c, I}}$ (i.e., the mass of the critical interaction cell). That provides alternative choice between two pairs of energy and length units which originate from thermodynamics normalized, either per particle, or per mass unit, respectively. However, at exact critical point $\left(\Delta \tau^{*}=0 ; \Delta \tilde{\rho}=0\right)$, the amount of matter $\frac{m_{\bar{p}}}{Z_{c}}$ is precisely contained within the microscopic interaction cell and the extensive nature of the fluid remains only characterized by $\frac{1}{Z_{c}}$, i.e., the particle number which fill this critical interaction cell. Along the critical isochore, $\frac{1}{Z_{c}}=$ constant is like a constant coordination number (of typical value $\sim 3.5$ ). Only $v_{c, I}=\left(\alpha_{c}\right)^{d}$ takes physical meaning in terms of the short ranged molecular interactions between the interacting $\frac{1}{Z_{c}}$ particles, leading to better understanding of the correlation length value when it is measured in unit of $\alpha_{c}$, i.e., in unit of the critical interaction cell size [28-30].

In such a description in terms of the properties of the critical interaction cell, it is then essential to note that the use of Eq. (60) gives unequivocal access to the two characteristics scale factors $Z_{c}$ and $Y_{c}$ of each one-component fluid belonging to the $\{1 f\}$-subclass of universality. $Z_{c}$ is then the first asymptotic scale factor, characteristic of the singular master behavior of the one-component fluid along the critical isotherm while the second scale factor $Y_{c}$, characteristic of the singular master behavior of the one-component fluid along the critical isochore, is

$$
Y_{c}=\gamma_{c}^{\prime} \frac{T_{c}}{p_{c}}-1
$$


In Eq. (64), $\gamma_{c}^{\prime} \frac{T_{c}}{p_{c}}$ is the usual critical Riedel factor (of typical value $\sim 5-6$ ). More precisely, $Y_{c}$ is here above defined from the limiting critical slope of the thermodynamic potential $4 \frac{p}{T}$, which is expressed in units of $\frac{k_{B}}{\left(\alpha_{c}\right)^{d}}=\frac{p_{c}}{T_{c}} . Y_{c}$ is then the single critical parameter only dependent on the selected critical length unit of Eq. (61), which introduces the single characteristic value of the entropy per particle ${ }^{5}$ at the critical point [46]. Therefore, the above master asymptotic behavior of the $\{1 f\}$-subclass assumes that all the thermodynamics properties of the interaction cell are master constants whatever the one-component fluid, except two of them, i.e., $Z_{c}$ and $Y_{c}$.

\section{Three Scale Factor Characterization of the Simple Fluid PAD}

The three scale factor set

$$
S_{S F, f}^{\{M R\}}=\left\{\vartheta ; g_{0} ; \psi_{\rho}\right\}
$$

characterizes each one-component fluid $f$ as a system satisfying the MR Eqs. (40), (41), and (42). This set is introduced through the following linearized asymptotic equations between theoretical and physical quantities:

$$
\begin{aligned}
t & =\vartheta \Delta \tau^{*} \\
h & =\psi_{\rho} \Delta \tilde{\mu} \\
\ell_{\mathrm{PAD}, \mathrm{th}}(t) & =g_{0} \xi_{\text {expt }}\left(\Delta \tau^{*}\right)
\end{aligned}
$$

Equivalently, the three scale factor set

$$
S_{S F, f}^{\{1 f\}}=\left\{Y_{c} ; \alpha_{c} ; Z_{c}\right\}
$$

characterizes each one-component fluid $f$ as a system satisfying the master description of Eqs. (50), (51), and (52). This set is obtained through the following similar linearized asymptotic equations between master and physical quantities:

$$
\begin{aligned}
\mathcal{T}^{*} & =Y_{c} \Delta \tau^{*} \\
\mathcal{H}^{*} & =\left(Z_{c}\right)^{-\frac{1}{2}} \Delta \tilde{\mu} \\
\ell^{*}\left(\mathcal{T}^{*}\right) & =\left(\alpha_{c}\right)^{-1} \xi_{\text {expt }}\left(\Delta \tau^{*}\right)
\end{aligned}
$$

The MR and master scale factors characteristics of each one-component fluid are then related by:

$$
\begin{aligned}
\vartheta\left(Y_{c}\right)^{-1} & =\Theta^{\{1 f\}} \\
g_{0} \alpha_{c} & =\mathbb{L}^{\{1 f\}} \\
\psi_{\rho}\left(Z_{c}\right)^{\frac{1}{2}} & =\Psi^{\{1 f\}}
\end{aligned}
$$

Equation (74) links $g_{0}$ defined for $\{t=0 ; h=0\}$ to $\alpha_{c}$ defined for $\left\{\Delta \tau^{*}=0 ; \Delta \tilde{\mu}=0\right\}$. Consequently, the MR, master and then physical PAD descriptions are interrelated through

\footnotetext{
4 The thermodynamic potential $\frac{p}{T}$ corresponds to the use of the Massieu form of the Grand Potential $p V$ when thermodynamics is normalized per unit volume.

5 The entropy per particle $s_{\bar{p}}$ is related to the temperature derivatives $\gamma^{\prime}=\left(\frac{\partial p}{\partial T}\right)_{v_{\bar{p}}}$ and $\delta^{\prime}=\left(\frac{\partial \mu_{\bar{p}}}{\partial T}\right)_{v_{\bar{p}}}$, which, at the critical point, lead to the following dimensionless equation $s_{\bar{p}, c}^{*}=Z_{c}\left(Y_{c}+1\right)-x_{\bar{p}, c}^{*}$, where $s_{\bar{p}, c}^{*}=\frac{s_{\bar{p}, c}}{k_{B}}$ and $x_{\bar{p}, c}^{*}=\frac{\delta_{c}^{\prime}}{k_{B}}$ are two particle quantities made dimensionless without reference to the physical scales $\alpha_{c}$ and $\left(\beta_{c}\right)^{-1}$.
} 
the following asymptotic equations between their corresponding fields:

$$
\begin{aligned}
t & =\vartheta \Delta \tau^{*}=\Theta^{\{1 f\}} Y_{c} \Delta \tau^{*} \\
h & =\psi_{\rho} \Delta \tilde{\mu}=\Psi^{\{1 f\}}\left(Z_{c}\right)^{-\frac{1}{2}} \Delta \tilde{\mu} \\
\ell_{\mathrm{PAD}, \text { th }}(t) & =g_{0} \xi_{\operatorname{expt}}\left(\Delta \tau^{*}\right)=\mathbb{L}^{\{1 f\}^{*}} \xi_{\text {expt }}\left(\Delta \tau^{*}\right)
\end{aligned}
$$

We note that, $\tilde{\chi}_{T \text {,expt }}=\left(\frac{\partial \Delta \tilde{\rho}}{\partial \Delta \tilde{\mu}}\right)_{\Delta \tau^{*}}=\left(\frac{\rho}{\rho_{c}}\right)^{2} p_{c} \kappa_{T, \text { expt }}$. As a result, along the critical isochore $\Delta \tilde{\rho}=0, \tilde{\chi}_{T \text {,expt }}\left(\Delta \tau^{*}\right) \equiv \kappa_{T \text {,expt }}^{*}\left(\Delta \tau^{*}\right)$ is then Ising-like similar to the theoretical susceptibility $\chi_{\text {th }}(t)=\left(\frac{\partial m_{t h}}{\partial h}\right)_{t}$, where $m_{\text {th }}=\left(\mathbb{L}^{\{1 f\}}\right)^{-d}\left(\psi_{\rho}\right)^{-1} \Delta \tilde{\rho}=$ $\left(\mathbb{L}^{\{1 f\}}\right)^{-d}\left(\Psi^{\{1 f\}}\right)^{-1} \mathcal{M}^{*}=\left(\mathbb{L}^{\{1 f\}}\right)^{-d}\left(\Psi^{\{1 f\}}\right)^{-1}\left(Z_{c}\right)^{\frac{1}{2}} \Delta \tilde{\rho}$. Accordingly, the asymptotic matching between MR and physical quantities of interest is provided by the equations:

$$
\begin{aligned}
\ell_{\mathrm{PAD}, \text { th }}(t) & =\mathbb{L}^{\{1 f\}} \xi_{\text {expt }}^{*}\left(\Delta \tau^{*}\right) \\
\chi_{\mathrm{PAD}, \text { th }}(t) & =\left(\mathbb{L}^{\{1 f\}}\right)^{-d}\left(\psi_{\rho}\right)^{-2} \kappa_{T, \text { expt }}^{*}\left(\Delta \tau^{*}\right) \\
m_{\mathrm{PAD}, \text { th }}(|t|) & =\left(\mathbb{L}^{\{1 f\}}\right)^{-d}\left(\psi_{\rho}\right)^{-1} \Delta \tilde{\rho}_{L V \text {,expt }}\left(\left|\Delta \tau^{*}\right|\right)
\end{aligned}
$$

while the equations:

$$
\begin{aligned}
\ell^{*}\left(\mathcal{T}^{*}\right) & =\xi_{\text {expt }}^{*}\left(\Delta \tau^{*}\right) \\
\mathcal{X}^{*}\left(\mathcal{T}^{*}\right) & =Z_{c} \kappa_{T, \text {expt }}^{*}\left(\Delta \tau^{*}\right) \\
\mathcal{M}\left(\left|\mathcal{T}^{*}\right|\right) & =\left(Z_{c}\right)^{\frac{1}{2}} \Delta \tilde{\rho}_{L V, \text { expt }}\left(\left|\Delta \tau^{*}\right|\right)
\end{aligned}
$$

provide similar asymptotic matching between master and physical quantities. Above matching equations are guarantees for uniqueness [7] of the length and energy units in the dimensionless singular behaviors of thermodynamic and correlations functions (whatever the one-component fluid).

Finally, the expected estimation of $S_{A, f}$ of Eq. (59) is obtained from the term to term matching in Eqs. (79), (80) and (83), (82), leading to:

$$
S_{A, f}=\left\{\begin{array}{l}
a_{\chi}^{+}=\vartheta^{\Delta} \mathbb{Z}_{\chi}^{1,+}=\left(Y_{c}\right)^{\Delta} \mathcal{Z}_{\chi}^{1,+} \\
\xi^{+}=\left(\mathbb{L}^{\{1 f\}}\right)^{-1} \vartheta^{-v}\left(\mathbb{Z}_{\xi}^{+}\right)^{-1}=\left(Y_{c}\right)^{-v} \mathcal{Z}_{\xi}^{+} \\
\Gamma^{+}=\left(\mathbb{L}^{\{1 f\}}\right)^{d}\left(\psi_{\rho}\right)^{2} \vartheta^{-\gamma}\left(\mathbb{Z}_{\chi}^{+}\right)^{-1}=\left(Z_{c}\right)^{-1}\left(Y_{c}\right)^{-\gamma} \mathcal{Z}_{\chi}^{+}
\end{array}\right\}
$$

We note the top-down hierarchy of Eqs. (85) that link $S_{A, f}$ and $\mathbb{S}_{A}^{\{M R\}}$. Top equation shows that $\vartheta$ characterizes the lowest order of the fluid confluent corrections to scaling in an unequivocal manner. Then medium equation defines the dimensionless number $\mathbb{L}^{\{1 f\}}$, which relates the microscopic length $\left(g_{0}\right)^{-1}$ and the thermodynamic length scale $\alpha_{c}$. Finally, bottom equation introduces the scale factor $\psi_{\rho}$ of the order parameter density. $\psi_{\rho}$ can be estimated using any leading amplitude chosen among the ones of the extensive singular properties, as here $\Gamma^{+}$in the isothermal compressibility case. When the dimensionless number $\mathbb{L}^{\{1 f\}}$ takes its master value of Eq. (49), the fluid scale factors $\vartheta$ and $\psi_{\rho}$ are then unequivocally related to $Y_{c}$ and $Z_{c}$, respectively, as shown by Eqs. (73) and (75). The correlation length is measured in units of $\alpha_{c}$ [39] and the thermodynamic energy densities are well measured in units of $\left[\beta_{c}\left(\alpha_{c}\right)^{d}\right]^{-1}=p_{c}[16,29,30]$, as previously expected. The top Eq. (85) shows that any first term of the confluent corrections to scaling is governed by a single characteristic parameter 
(i.e., $Y_{c}$ ) of the critical interaction cell. In other words, along the critical isochore, the firstorder contribution of the confluent corrections of any fluid is well due to the expected master properties of its critical interaction volume, only characterized by the two dimensionless numbers $Y_{c}$ and $Z_{c}$ (see also Ref. [46]).

In addition, Eqs. (44) to (46) lead to:

$$
\begin{aligned}
B & =\left(\mathbb{L}^{\{1 f\}}\right)^{d} \psi_{\rho} \vartheta^{\beta} \mathbb{Z}_{M}=\left(Z_{c}\right)^{-\frac{1}{2}}\left(Y_{c}\right)^{\beta} \mathcal{Z}_{M} \\
a_{\xi}^{+} & =\vartheta^{\Delta} \mathbb{Z}_{\xi}^{1,+}=\left(Y_{c}\right)^{\Delta} \mathcal{Z}_{\xi}^{1,+} \\
a_{M} & =\vartheta^{\Delta} \mathbb{Z}_{M}^{1}=\left(Y_{c}\right)^{\Delta} \mathcal{Z}_{M}^{1}
\end{aligned}
$$

The estimates of the amplitudes involved in Eqs. (55) to (57) are thus closed for any fluid case. The value of the dimensional amplitude $\xi_{0}^{+}$is,

$$
\xi_{0}^{+}=\alpha_{c} \xi^{+}
$$

It is also convenient to add the following amplitude estimation for the specific heat case

$$
A^{+}=\left(\mathbb{L}^{\{1 f\}}\right)^{d} \vartheta^{2-\alpha} \alpha \mathbb{Z}_{C}^{+}=\left(Y_{c}\right)^{2-\alpha} \mathcal{Z}_{C}^{+}
$$

in order to control the Ising-like universal values of $R_{C}=\frac{A^{+} \Gamma^{+}}{B^{2}}, R_{\xi}^{+}=\left(A^{+}\right)^{\frac{1}{d}} \xi^{+}$and Eqs. (44) to (46) only using the minimal set of four critical coordinates of Eq. (60).

In an alternative manner, we recall that the knowledge of $S_{S F, f}^{\{M R\}}$ of Eq. (65), or $S_{S F, f}^{\{M R\}}$ of Eq. (69), equivalently, provides also access to the asymptotic fluid characterization exchanging the unknown leading amplitude set $S_{A, f}$ by the leading prefactor set $S_{1 C P, \mathcal{L}, f}^{\{M R\}}=$ $\left\{\vartheta ; \mathbb{L}_{0, \mathcal{L}}^{*} ; \mathbb{X}_{0, \mathcal{L}}^{*}\right\}$ of Eq. (17), when the identity $\vartheta \equiv \vartheta_{\mathcal{L}}$ is assumed correct over an extended temperature range. In such a case, the respective estimations of the prefactors $\mathbb{L}_{0, \mathcal{L}}^{*}, \mathbb{X}_{0, \mathcal{L}}^{*}$, and $\mathbb{M}_{0, \mathcal{L}}^{*}$ by Eqs. (24), (36), and (37) are made to conformity to the two-scale-factor universality, while the following equation for the prefactor $\mathbb{C}_{0, \mathcal{L}}^{*}$ in the specific heat case [11]

$$
\begin{aligned}
\mathbb{C}_{0, \mathcal{L}}^{*} & =\left(\mathbb{L}^{\{1 f\}}\right)^{d} \vartheta^{2-\alpha} \\
& =\left(\mathbb{L}^{\{1 f\}}\right)^{d}\left(\Theta^{\{1 f\}}\right)^{2-\alpha}\left(Y_{c}\right)^{2-\alpha}
\end{aligned}
$$

guaranties the validity of the hyperscaling universal combination $R_{\xi}^{+}$, through the identity $\left(\mathbb{C}_{0, \mathcal{L}}^{*}\right)^{\frac{1}{d}} \mathbb{L}_{0, \mathcal{L}}^{*} \equiv 1$, which complements the identity of Eq. (16) (or $\mathbb{C}_{0, \mathcal{L}}^{*} \frac{\mathbb{X}_{0, \mathcal{L}}^{*}}{\left(\mathbb{M}_{0, \mathcal{L}}^{*}\right)^{2}}=1$, equivalently).

\section{Extension of the Ising-Like Preasymptotic Domain of a Simple Fluid $f$}

The value of $\vartheta$ enables the estimation of $\mathcal{L}_{\mathrm{PAD}}^{f}$, which fixes the physical extension of the Ising-like preasymptotic domain [9] from the equation

$$
\mathcal{L}_{\mathrm{PAD}}^{f} \simeq \frac{\mathcal{L}_{\mathrm{PAD}}^{\mathrm{Ising}}}{\vartheta(f)}
$$

This important result can be recovered from the value of $Y_{c}$ and Eq. (54), using then the equation

$$
\mathcal{L}_{\mathrm{PAD}}^{f} \simeq \frac{\mathcal{L}_{\mathrm{PAD}}^{\{1 f\}}}{Y_{c}(f)}
$$


The crucial problem to define the temperature range of validity of Eqs. (55) to (57) is now solved quantitatively. Indeed, using Eq. (54) and a typical value of $Y_{c}(f) \sim 4-5$ for $f$, the validity range of Eqs. (55) to (57) is $\Delta \tau^{*} \lesssim \mathcal{L}_{\mathrm{PAD}}^{f} \sim 10^{-4}$, typically.

However, in such a small temperature range, the experimental control of the above three parameters characterization of a simple fluid cannot be easily performed since accurate measurements of the singular properties are generally made in a temperature range which does not reach this small Ising-like preasymptotic domain. As a result, the Ising-like equivalence between the amplitudes and the scale factors is not obtained with the required precision from the fits of the experimental results.

\section{Xenon Case}

\section{Xenon Critical Coordinates}

The xenon critical temperature was fixed to the value $T_{c}=289.733 \pm 0.002 \mathrm{~K}$ recently recommended by Gillis et al $[47,48]$ from their critical temperature determination of the stirred xenon filling its acoustic resonator cell submitted to a ramp of temperature downward (in this experiment the absolute temperature precision is $\pm 15 \mathrm{mK}$ from reference to the ITS- 90 temperature scale). This value agrees with the two respective values $T_{c}=289.731 \pm 0.0053 \mathrm{~K}$ and $T_{c}=289.734 \pm 0.003 \mathrm{~K}$ measured (with an absolute precision of $\pm 50 \mathrm{mK}$ ) by Berg and al [49] from observation of the vapor-liquid meniscus appearance and disappearance in the "Critical Viscosity of Xenon (CVX)" experiment. These central values, and their relative uncertainties essentially due to the thermostat temperature control, compare well with $T_{c}=289.740 \pm 0.003 \mathrm{~K}$ obtained by Schneider et al from $p V T$ measurements [50,51] and density measurements of coexisting liquid and vapor phases [52,53]. This latter value was generally used as a xenon critical temperature in previous review analyzes $[28,37,42]$ using the ITS-68 temperature scale. Indeed, the agreement was noticeable with $T_{c}=289.747 \pm$ $0.010 \mathrm{~K}$ obtained by Cannell and Benedek [54] from measurements of Brillouin spectrum, and $T_{c}=289.736 \pm 0.002 \mathrm{~K}$ obtained by Smith et al [55] from light scattering intensity measurements. However, the Gillis et al's central value disagrees with some other values of similar relative precision (as for example: $T_{c}=289.765 \pm 0.005 \mathrm{~K}$ from Baidakov et al's [56,57]; $T_{c}=289.790 \pm 0.001 \mathrm{~K}$ from Güttinger and Cannell [20]; $T_{c}=289.752 \pm 0.001 \mathrm{~K}$ and $T_{c}=289.789 \pm 0.002 \mathrm{~K}$ from Balzarini et al's [34]).

Our calculated critical pressure $p_{c}=5.84007 \pm 0.00050 \mathrm{MPa}$ accounts for the thermodynamic continuity on pressure measurements crossing the critical temperature along the critical isochore and assumes that the Habgood and Schneider's isotherm $p(\rho)$ at $\left(T_{c}\right)_{H S}=(273.15+16.59) \mathrm{K}$ corresponds to the critical isotherm of xenon.

Our selected value of $\rho_{c}=1113 \mathrm{~kg} \mathrm{~m}^{-3}$ has an uncertainty of $\pm 5 \mathrm{~kg} \mathrm{~m}^{-3}(\sim \pm 0.5 \%)$, which accounts for the $\rho_{c}$ values of Schneider et al's $\left(\rho_{c}=1105 \pm n \cdot a \cdot \mathrm{kg} \mathrm{m}^{-3}\right.$ and $\left.\rho_{c}=1099 \pm n \cdot a \cdot \mathrm{kg} \mathrm{m}^{-3}\right)$ [50-53], Cornfeld and Carr's $\left(\rho_{c}=1111.2_{-3.4}^{+1.9} \mathrm{~kg} \mathrm{~m}^{-3}\right.$ for three different estimations) [35], Baidakov et al's $\left(\rho_{c}=1112.8 \pm n \cdot a \cdot \mathrm{kg} \mathrm{m}^{-3}\right)$ [56], and Balzarini et al's $\left(\rho_{c}=1099 \pm\right.$ n.a. $\mathrm{kg} \mathrm{m}^{-3}[36], \rho_{c}=1116.0 \pm 1.7 \mathrm{~kg} \mathrm{~m}^{-3}$ and $\rho_{c}=1114.7 \pm 1.7 \mathrm{~kg} \mathrm{~m}^{-3}$ [34]). All the above determinations of the critical density were obtained without any measurable curvature effect of the rectilinear density diameter.

The value $\gamma_{c}^{\prime}=0.1197 \pm 0.0006 \mathrm{MPa} \mathrm{K}^{-1}(\sim \pm 0.5 \%)$ was estimated from the joint analysis of the $p V T$ measurements of Habgood and Schneider [50,51] and Michels et al [58], to account for the small differences on the critical density values. As a matter of fact, in spite of numerous values reported in the seventies literature, the determination of this finite critical 
derivative was never accurately analysed in xenon case [28]. We recall that, at the late sixties, the knowledge of the derivative $\left(\frac{\partial p}{\partial T}\right)_{\rho}$ in the vicinity of the critical point was mandatory needed when the objectives were to define the scaled forms of the equation of state (see Refs. $[43,59-63])$ and to test their related computations of the thermophysical property singularities (since the dimensionless quantity $\frac{T}{p_{c}}\left(\frac{\partial p}{\partial T}\right)_{\rho_{c}}$-the so-called Riedel factor-appears in many thermodynamic relations). In xenon case, the dimensionless value $\frac{T_{c}}{p_{c}} \gamma_{c}^{\prime}=6.02$ was initially obtained by Vicentini-Missoni et al [60] from their fitting of the $p V T$ measurements of Habgood and Schneider (with $p_{c}=5.83 \mathrm{MPa}, T_{c}=289.75 \mathrm{~K}$, and $\rho_{c}=1110 \mathrm{~kg} \mathrm{~m}^{-3}$, as xenon critical coordinates). The related uncertainty on the dimensional value $\gamma_{c}^{\prime}=0.1211 \mathrm{MPa} \mathrm{K}^{-1}$ was not given, in spite of the fact that this value was higher $(\sim+1.8 \%)$ than the one $\gamma_{c}^{\prime}=0.1189 \mathrm{MPaK}^{-1}$ initially found by Habgood and Schneider from a self-consistent analysis of the derivative $\left(\frac{\partial p}{\partial T}\right)_{\rho}$ graphically deduced from their $p V T$ measurements. Subsequently, several published values (such as $\gamma_{c}^{\prime}=0.11916 \mathrm{MPa} \mathrm{K}^{-1}$ from Cannell and Benedek [54], $\gamma_{c}^{\prime}=0.12027 \mathrm{MPaK}^{-1}$ from Smith et al [55], $\gamma_{c}^{\prime}=0.1196 \mathrm{MPa} \mathrm{K}^{-1}$ from Swinney and Henry [64], $\gamma_{c}^{\prime}=0.1192 \pm 0.0012 \mathrm{MPa} \mathrm{K}^{-1}$ from Garrabos [28]) were obtained from these Habgood and Schneider's data source and same xenon critical parameters. On the other hand, Badaikov et al have determined two values, $\gamma_{c}^{\prime}=0.11865 \mathrm{MPa} \mathrm{K}^{-1}$ [56], and $\gamma_{c}^{\prime}=0.11977 \mathrm{MPa} \mathrm{K}^{-1}$ [57], from their vapor pressure data below $T_{c}$. Berg et al $[49,65]$ have used the dimensionless value $\frac{T_{c}}{p_{c}} \gamma_{c}^{\prime}=5.65$ (with an uncertainty of $\pm 2.9 \%$ ), in their viscosity data analysis of the CVX experiment with $\rho_{c}=1110 \mathrm{~kg} \mathrm{~m}^{-3}$. Their related value $\gamma_{c}^{\prime}=0.113686 \mathrm{MPa} \mathrm{K}^{-1}$ is significantly lower $(\simeq-5 . \%)$ than our selected value $\gamma_{c}^{\prime}=0.1197 \mathrm{MPaK}^{-1}$. More recently, Gillis et al, selecting the "highest" value $\rho_{c}=1116 \mathrm{~kg} \mathrm{~m}^{-3}$ of the critical density, have used the dimensionless value $\frac{T_{c}}{p_{c}} \gamma_{c}^{\prime}=5.9253$ calculated by Swinney and Henry for the presumable critical isochore $\rho=1110 \mathrm{~kg} \mathrm{~m}^{-3}$, attributing then an uncertainty of $0.2 \%$ on the corresponding value $\gamma_{c}^{\prime}=0.1195 \mathrm{MPa} \mathrm{K}^{-1}$. Therefore, even today, the largest uncertainty in the xenon critical coordinates of Eq. (92) comes from the determination of $\gamma_{c}^{\prime}$, which is then dependent of the selected value for the critical density. In the future, a better estimation of $\gamma_{c}^{\prime}$ needs that the two derivatives $\left(\frac{\partial p}{\partial T}\right)_{\rho}$ and $\left[\frac{\partial}{\partial \rho}\left(\frac{\partial p}{\partial T}\right)_{\rho}\right]_{T}$ are determined simultaneously in the vicinity of the critical point in order to correctly account for the contribution of the relative uncertainty in the $\rho_{c}$ value.

\section{Xenon Critical Parameters}

The selected critical coordinates of xenon are:

$$
\begin{aligned}
T_{c} & =289.733 \mathrm{~K}, \\
p_{c} & =5.84007 \mathrm{MPa}, \\
\rho_{c} & =1113 \mathrm{~kg} \mathrm{~m}^{-3} \text { or } v_{\bar{p}, c}=0.19596 \mathrm{~nm}^{3}, \\
\gamma_{c}^{\prime} & =0.1197 \mathrm{MPa} \mathrm{K}^{-1}, \\
m_{\bar{p}} & =2.1805 \times 10^{-25} \mathrm{~kg} .
\end{aligned}
$$

Two remarks can be formulated.

(i) The values of Eq. (92) are in remarkable agreement with the ones defined by Gillis et al [47] in their analysis of the sound attenuation (in the frequency range $100<f(\mathrm{~Hz})<$ 7500) by thermoacoustic layers between solid surfaces and xenon at critical density. 
Table 1 Characteristics parameters in the case of xenon

\begin{tabular}{|c|c|c|c|c|c|c|c|}
\hline \multirow{2}{*}{$\frac{\left(\beta_{c}\right)^{-1}}{S_{S F, \mathrm{Xe}}^{\{1 f\}}}$} & \multicolumn{7}{|c|}{$4.0003 \times 10^{-21} \mathrm{~J}$} \\
\hline & $\begin{array}{l}\text { Master scale } \\
\text { factors }\end{array}$ & $S_{S F, \mathrm{Xe}}^{\{M R\}}$ & $\begin{array}{l}\text { MR scale } \\
\text { factors }\end{array}$ & $S_{A, \mathrm{Xe}}$ & $\begin{array}{l}\text { Physical } \\
\text { amplitudes }\end{array}$ & $S_{1 C, \mathcal{L}, \mathrm{Xe}}^{\{M R\}}$ & MR prefactors \\
\hline$Y_{c}$ & 4.93846 & $\vartheta$ & 0.0211752 & $a_{\chi}^{+}$ & 1.23709 & $\vartheta_{\mathcal{L}}$ & 0.0211752 \\
\hline$\alpha_{c}$ & $0.881498 \mathrm{~nm}$ & $g_{0}$ & $29.0245 \mathrm{~nm}^{-1}$ & $\xi^{+}$ & 0.209338 & $\mathbb{L}_{0, \mathcal{L}}^{*}$ & 0.444008 \\
\hline \multirow[t]{7}{*}{$Z_{c}$} & 0.28602 & $\psi_{\rho}$ & $\begin{array}{c}3.28165 \times \\
10^{-4}\end{array}$ & $\Gamma^{+}$ & 0.0578238 & $\mathbb{X}_{0, \mathcal{L}}^{*}$ & 0.21454 \\
\hline & \multicolumn{7}{|c|}{ Auxiliary } \\
\hline & & & & $B$ & 1.46762 & $\mathbb{M}_{0, \mathcal{L}}^{*}$ & 1.56555 \\
\hline & & & & $a_{\xi}^{+}$ & 0.840217 & & \\
\hline & & & & $a_{M}$ & 1.11338 & & \\
\hline & & & & $A^{+}$ & 2.13835 & $\mathbb{C}_{0, \mathcal{L}}^{*}$ & 11.424187 \\
\hline & & & & $\xi_{0}^{+}$ & $0.184531 \mathrm{~nm}$ & & \\
\hline
\end{tabular}

(ii) The values of Eq. (92) are of basic interest using the scaled forms of the equation of state of xenon $[43,59-63,66]$. Especially in the linear-model parametric equation of state $[61,63]$ and the Ho and Lister's [61] restricted cubic model of the equation of state, the singular behavior of each fluid is characterized by only two dimensionless numbers $(k$ and $a$ in standard notations). Now we are able [11] to estimate the xenon parameters $k$ and $a$ only from our above values of the critical point coordinates. However, we recall that these parametric models are not quantitatively exact in regards to the Ising-like universal combinations of the leading amplitudes [67].

Using all the above equations given in this Appendix 1, we can estimate the related values of the xenon characteristic parameters given in Table 1, noting finally that the Ising-like preasymptotic domain of xenon corresponds to the temperature range:

$$
T-T_{c} \lesssim T_{c} \mathcal{L}_{\mathrm{PAD}}^{\mathrm{Xe}}=26 \mathrm{mK}
$$

\section{Appendix 2: Effective Power Law Analysis}

To replace the arbitrary truncated Wegner-like expansion of Eq. (25), a useful simple function to fit the isothermal compressibility data measured at finite distance to the critical temperature along the critical isochore of xenon is the pure power law:

$$
\kappa_{T, \mathrm{fit}}^{*}=\Gamma_{e, \mathrm{fit}}^{+}\left(\Delta \tau^{*}\right)^{-\gamma_{e, \mathrm{fit}}}
$$

with adjustable non-Ising exponent $\gamma_{e}$,fit and effective amplitude $\Gamma_{e}^{+}$,fit . The values of $\gamma_{e}$,fit and $\Gamma_{e}^{+}$,fit are then associated to the limited experimental temperature range $\mathcal{L}_{\mathrm{PAD}}^{\mathrm{Xe}}<\Delta \tau_{\text {min }}^{*} \leq$ $\Delta \tau^{*} \leq \Delta \tau_{\max }^{*}$ of the fit. For example, Güttinger and Cannell have claimed that the correction to scaling terms are important by demonstrating in Fig. 2 of Ref. [20], that their susceptibility measurements of high relative precision $(\sim \pm 0.2 \%)$ deviate systematically from Eq. (94) where $\gamma_{e, \mathrm{GC}}=1.206$ and $\Gamma_{e, \mathrm{GC}}^{+}=0.6390 \chi_{T, \rho}\left(\Delta \tau_{o r}^{*}\right) \frac{p_{c, \mathrm{GC}}\left(T_{c}\right)^{-1.206}}{\left(\rho_{c, \mathrm{GC}}\right)^{2}}=0.07867$, with $\rho_{c, \mathrm{GC}}=1110 \mathrm{~kg} \mathrm{~m}^{-3}$ and $p_{c, \mathrm{GC}}=5.84 \mathrm{MPa}$. Looking carefully to their figure, we 
note that $\gamma_{e, \mathrm{GC}} \simeq 1.206$ corresponds to the slope of the tangent line to the experimental behavior at the relative temperature distance $\Delta \tau_{\gamma_{e, \mathrm{GC}}=1.206}^{*} \simeq 4.35 \times 10^{-3}$, i.e., the local temperature $T \simeq T_{c}+1.25 \mathrm{~K}$ where the deviation curve shows an extrema (around $-4 \%$ ). Hereafter, we have reanalyzed this result considering the isothermal compressibility data calculated from the equation $\kappa_{T, \text { expt }}^{*}=\chi_{T, \rho, \operatorname{expt}} \frac{p_{c}}{\left(\rho_{c}\right)^{2}} \chi_{T, \rho}\left(\Delta \tau_{o r}^{*}\right)$, with $\rho_{c}=1113 \mathrm{~kg} \mathrm{~m}^{-3}$ and $p_{c}=5.84007 \mathrm{MPa}$. Using Eq. (94), we have then fitted the twelve data covering the restricted temperature range $9.115 \times 10^{-4} \leq \Delta \tau^{*} \leq 1.95 \times 10^{-2}\left(0.26 \mathrm{~K} \leq T-T_{c} \leq 5.65 \mathrm{~K}\right)$ defined by the segment labeled FIT and the pale grey area in Fig. 4. The mean (geometrical) value $\left\langle\Delta \tau_{\text {fit }}^{*}\right\rangle=\sqrt{\Delta \tau_{\min }^{*} \Delta \tau_{\max }^{*}}=4.215 \times 10^{-3}$ of this selected temperature range is close to the one of the residual extrema observed in Fig. 4. As expected, our fitting result

$$
\kappa_{T, \text { fit }}^{*}=0.07551466\left(\Delta \tau^{*}\right)^{-1.205879}
$$

shows an excellent agreement with the Güttinger and Cannell's result (the amplitude difference accounting for the $\simeq-4 \%$ deviation noted above). We note the significant difference between the above value $\Gamma_{e}^{+}$,fit $=0.07551466$ of the effective amplitude and our previous calculated value $\Gamma^{+}=0.0578238$ [see Eq. (85)] of the true asymptotic amplitude. In Fig. 4 , we have reported the residuals $\% R\left(\kappa_{T}^{*}\right)=100\left(\frac{\kappa_{T}^{*}}{\kappa_{T, \text { fit }}^{*}}-1\right)$ (expressed in $\%$ ) for all the data, enlightening the data dispersion at the $\pm 1 \%$ level in the blue grey area, which corresponds to our restricted fitted range (for easy link with our previous figures, the lower and upper horizontal axes are labeled in a similar manner). Then, our Fig. 4 complements Fig. 2 of Ref. [20] after a $-4 \%$ zero shift in the vertical axis and a magnification of the high relative precision $(\sim 0.2 \%)$ of the measurements.

However, it is essential to recall that the true experimental uncertainty of the amplitude value $\Gamma_{e \text {,fit }}=0.07551466$ mainly remains related to the error-bar $(\sim \pm 1.5 \%)$ on the calibration of the susceptibility data. To illustrate some other secondary effects, we have also estimated the residuals for the fits that use Eq. (25) with $\gamma=1.241, \Delta=0.496$, and the following three sets of parameters

(i) $\Gamma^{+}=0.0577, a_{1 \chi}^{+}=1.29, a_{2 \chi}^{+}=-1.59$, and $a_{3 \chi}^{+}=1.9$, i.e., the best fitting parameters obtained by Güttinger and Cannell with $\rho_{c}, \mathrm{GC}=1110 \mathrm{~kg} \mathrm{~m}^{-3}$, which corresponds to the dotted black curve labeled $\mathrm{GC}_{4}$ in Fig. 4;

(ii) $\Gamma^{+}=0.0577\left(\frac{\rho_{c}}{\rho_{c, \mathrm{GC}}}\right)^{2}$ and same values of $a_{1 \chi}^{+}=1.29, a_{2 \chi}^{+}=-1.59$, and $a_{3 \chi}^{+}=1.9$, leading to the full red curve labeled 1 , which shows the effect of our critical density value;

(iii) the latter parameter set and a shift of $0.5 \mathrm{mK}$ in $T_{c}$, illustrated by the dotted pink curve labeled 2, which accounts for the realistic increase of the experimental uncertainty near $T_{c}$.

The relative differences between curves 1 and 2 increase approaching the critical temperature. However this difference disappears in our fitted temperature range. The shape and amplitude differences between curves 1 and $\mathrm{GC}_{4}$ combine the effects due to the respective critical density values and the shift of $0.5 \mathrm{mK}$ in $T_{c}$, but without modification of the temperature where occurs the extrema. Finally, in Fig. 4, the well-defined horizontal coordinate of the extrema of the deviation curves is the most important consequence of the high relative precision of the Güttinger and Cannell's measurements. That demonstrates that the precise value (here $\left.\gamma_{e, \text { fit }}=1.205879\right)$ of the effective exponent can be measured at a well-defined temperature distance to $T_{c}$ (here $\simeq T_{c}+1.26 \mathrm{~K}$ ).

Now, we can refine the above power law analysis, accounting thus for the theoretical effective behavior $\mathbb{Z}_{\chi, e}^{+}\left(\gamma_{e, \text { th }}\right)$ of Fig. 1. The condition $\gamma_{e, \text { th }}=1.205879$ is obtained at 


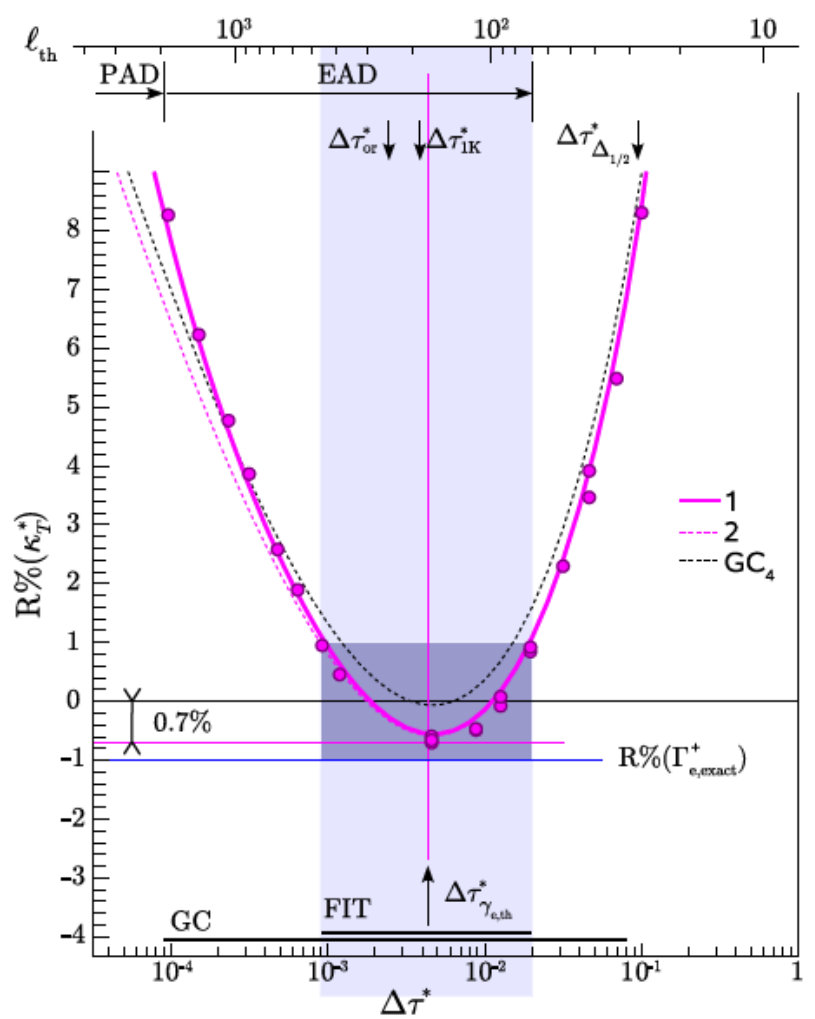

Fig. 4 Residuals $R \%\left(\kappa_{T}^{*}\right)$ (expressed in \%) of $\kappa_{T}^{*}$ data from the fitting result $\kappa_{T \text {, fit }}^{*}=$ $0.07551466\left(\Delta \tau^{*}\right)^{-1.205879}$ obtained over the limited temperature range illustrated by the segment labeled FIT (see the pale grey area). The $1 \%$ error-bar of the fit corresponds to the grey-blue area (see text). Segment labeled GC: full experimental temperature range of Ref. [20]. Red (full) circles: experimental data points obtained from Ref. [20], using $\rho_{c}=1113 \mathrm{~kg} \mathrm{~m}^{-3}$. Pink (full) curve labeled 1: Eq. (25), with $\rho_{c}=1113 \mathrm{~kg} \mathrm{~m}^{-3}$ and a $T_{c}$ shift of $0.5 \mathrm{mK}$. Pink (dotted) curve labeled 2: Eq. (25), with $\rho_{c}=1113 \mathrm{~kg} \mathrm{~m}^{-3}$. Black (dotted) curve labeled $\mathrm{GC}_{4}$ : Eq. (25) from Ref. [20], with $\rho_{c, \mathrm{GC}}=1110 \mathrm{~kg} \mathrm{~m}^{-3}$. Horizontal and vertical (pink) lines from reference to the local value $\Gamma_{e, \text { env }}^{+}=0.074986$ at $\Delta \tau_{\gamma_{e, \text { th }}}^{*}=4.347 \times 10^{-3}$ where $\gamma_{e, \text { th }}=\gamma_{e, \text { fit }}=1.205879$ (see text). Other symbols, marks and labels: see text and previous figures (Color figure online)

$t_{\gamma_{e, \text { th }}=1.205879}=9.159 \times 10^{-5}$, where $\mathbb{Z}_{\chi, \gamma_{e, \text { th }}}^{+}=0.396926$ (see the point $\mathrm{T}$ in Fig. 1). Using then $t=\vartheta \Delta \tau^{*}$ [Eq. (66) ] with $\vartheta \stackrel{\chi}{=} 0.0211752$ defined in $\S 2$, we expect to observe $\gamma_{e \text {,fit }}=1.205879$ at $\Delta \tau_{\gamma_{e, \text { th }}^{*}=1.205879}^{*}=4.347 \times 10^{-3}$ (i.e., $T-T_{c} \simeq+1.26 \mathrm{~K}$ ), in excellent agreement with the experimental position of the extrema of the deviation curve 1 in Fig. 4.

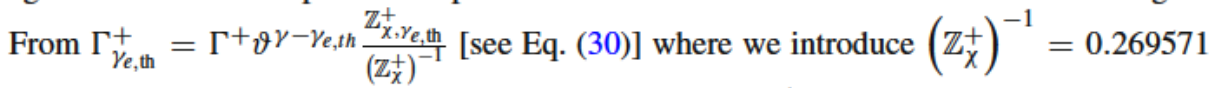
and $\Gamma^{+}=0.057824$ previously defined in $\S 1$, we obtain $\Gamma_{\gamma e \text {,th }=1.205879}^{+}=0.0747481$, i.e., a value only $\sim 1 \%$ lower than $\Gamma_{e}^{+}$,fit . However, rewriting then Eq. (31) in the following form

$$
\vartheta_{e, \text { fit }}=\left[\frac{\left(\mathbb{Z}_{\chi}^{+}\right)^{-1}}{\mathbb{Z}_{\chi}^{+}, \gamma_{e, t h}} \times \frac{\Gamma_{e, \text { fit }}^{+}}{\Gamma^{+}}\right]^{\frac{1}{\gamma-\gamma_{e, \text { fit }}}}
$$


we obtain $\vartheta_{e \text {,fit }}=0.028466$, i.e., a value $\sim 35 \%$ higher than our asymptotic value $\vartheta=$ 0.0211752. Such an important discrepancy needs to complement our understanding of the role of the experimental uncertainty on $\Gamma_{e, \text { fit }}^{+}$in Eq. (96).

Indeed, a fitting procedure where the effective exponent and amplitude are free in minimizing the mean deviations over a finite temperature range remains incomplete. We must then introduce the local tangent envelope of the $\kappa_{T}^{*}\left(\Delta \tau^{*}\right)$-curve defined by the equation $\kappa_{T, \text { env }}^{*}=\Gamma_{e, \text { env }}^{+}\left(\Delta \tau^{*}\right)^{-\gamma_{e} \text {,fit }}$, where $\Gamma_{e, \text { env }}^{+}$is such as $\Gamma_{e, \text { fit }}^{+} \neq \Gamma_{e, \text { env }}^{+}$. For example, in Fig. 4 , we can observe that the true tangent (pink) line of slope $\gamma_{e}$,fit $=1.205879$ has an amplitude $\simeq 0.7 \%$ lower than the amplitude $\Gamma_{e}^{+}$,fit , thanks to the $0.2 \%$ relative precision of the Güttinger and Cannell measurements. We obtain $\Gamma_{e, \text { env }}^{+}=0.074986$ (see the point P in Fig. 1). More generally, from Eq. (96) and a careful analysis of the fit deviations, we can always write $\Gamma_{e, \text { env }}^{+}=\Gamma_{e, \text { fit }}^{+}\left(1+\delta \Gamma_{e, \text { fit }}\right)$ and then define the value $\vartheta_{e \text {, env }}$ related to the tangent envelope by the equation

$$
\vartheta_{e, \mathrm{env}}=\vartheta_{e, \mathrm{fit}}\left(\frac{\Gamma_{e, \mathrm{env}}^{+}}{\Gamma_{e, \mathrm{fit}}^{+}}\right)^{\frac{1}{\gamma-\gamma_{e, \mathrm{fit}}}} \simeq \vartheta_{e, \mathrm{fit}}\left(1+\frac{\delta \Gamma_{e, \mathrm{fit}}}{\gamma-\gamma_{e, \mathrm{fit}}}\right)
$$

From the Güttinger and Cannell's results of Fig. 4 where $\delta \Gamma_{e, \text { fit }} \simeq-0.007$ and $\vartheta_{e, \text { fit }} \simeq$ 0.028466 , we obtain $\vartheta_{e, \text { env }} \simeq 0.022556$ which is now in better agreement $(+6.5 \%)$ with our asymptotic value $\vartheta \simeq 0.211752$. In Fig. 2 a, the effective slope $\gamma_{e \text {,fit }}=1.205879$ should be observed at $\Delta \tau_{\gamma_{e, t h}=\gamma_{e, \text { fit }}}^{*}=4.347 \times 10^{-3}$ (see the vertical dotted pink line), while the values of $\vartheta_{\mathcal{L}}=\vartheta_{e, \text { cor }}=0.022556$ [see Eq. (97)] and $R \%\left(\vartheta_{e \text {,cor }}\right)_{\vartheta}=+6.5 \%$ (see Eq. (102)) are represented by the full blue points in parts (b) and (c) of Fig. 2, respectively. We note that the condition $\gamma_{e \text {,th }}(t)=\gamma_{e \text {,expt }}\left(\Delta \tau^{*}\right)$ is satisfied for $t \cong 9.08 \times 10^{-5}$, where $\ell_{\text {th }} \cong 174.7 \cong 6.77 \mathbb{L}^{\{1 f\}}$ (or equivalently, for $T-T_{c} \cong 1.26 \mathrm{~K}$, where $\xi_{\text {expt }} \cong 6.77 \alpha_{c}$ ), i.e., a correlation length value within our Ising-like extended asymptotic domain defined by the condition $\ell_{\mathrm{th}}(t) \gtrsim 3 \mathbb{L}^{\{1 f\}} \simeq 75$ (or $\frac{\xi_{\text {expt }}}{\alpha_{c}} \gtrsim 2.5-3$, as analyzed in Refs. [32,39]). This accurate tangent description of the singular behavior of the isothermal compressibility of xenon is one major point of interest of the Güttinger and Cannell's results to validate the one-parameter crossover modeling predicted by the massive renormalization scheme beyond the Ising-like preasymptotic domain.

Since the Güttinger and Cannell's results of highly relative precision $(\sim 0.2 \%)$ have illustrated the significant role of the exponent difference $\gamma-\gamma_{e}$, we can also estimate the related temperature effect of the uncertainty on the asymptotic value of $\vartheta$ (as calculated in $\S 1$ ). We thus consider an effective fitting procedure which provides the values of $\gamma_{e}, \Gamma_{e}^{+}$, and $\Delta \tau_{e}^{*}$, where it is assumed that $\Gamma_{e}^{+}$is affected by a relative error-value $\delta \Gamma_{e}$, such as

$$
\Gamma_{e, \text { exact }}^{+}=\Gamma_{e}^{+}\left(1+\delta \Gamma_{e}\right)
$$

In Eq. (98), $\Gamma_{e, \text { exact }}^{+}$is de facto the calculated value $\left(\Gamma_{e, \text { exact }}^{+} \equiv \Gamma_{\gamma_{e, \mathrm{th}}=1.205879}^{+}=0.0747481\right)$ when $\vartheta$ is defined such that

$$
\vartheta=\left[\frac{\left(\mathbb{Z}_{\chi}^{+}\right)^{-1}}{\mathbb{Z}_{\chi, e}^{+}} \times \frac{\Gamma_{e, \text { exact }}^{+}}{\Gamma^{+}}\right]^{\frac{1}{\gamma-\gamma_{e}}}
$$

In Eq. (99), the value of $\mathbb{Z}_{\chi}^{+}, \vartheta, \Gamma^{+}, \gamma_{e}$,th $\equiv \gamma_{e}$, and $\mathbb{Z}_{\chi, e}^{+}\left(\gamma_{e, \text { th }}\right)$ are known with zero uncertainty (from the mean crossover function $\chi_{\text {th }}(t)$ given in [9]). Using then Eq. (94) and (99), we obtain 


$$
\vartheta_{e}=\vartheta\left(\frac{1}{1+\delta \Gamma_{e}}\right)^{\frac{1}{\gamma-\gamma_{e}}}
$$

For each value $\gamma_{e}=\gamma_{e}$, th estimated at $\Delta \tau_{\gamma_{e, \text { th }}}^{*}=\frac{t_{\gamma_{e, \text { th }}}}{\vartheta}$ with $\vartheta=0.0211752$, we can easily calculate the isocline $\vartheta_{e, \delta \Gamma_{e}}\left(\Delta \tau^{*}\right)$ at constant (small) value of $\delta \Gamma_{e}$, using the following approximation

$$
\vartheta_{e, \delta \Gamma_{e}}\left(\Delta \tau^{*}\right) \simeq \vartheta\left[1-\frac{\delta \Gamma_{e}}{\gamma-\gamma_{e}}\right]
$$

The corresponding isocline of the residuals is thus

$$
r \%\left(\vartheta_{e, \delta \Gamma_{e}}\right)=100\left(\frac{\vartheta_{e, \delta \Gamma_{e}}}{\vartheta}-1\right)=-\frac{\delta \Gamma_{e}}{\gamma-\gamma_{e}}
$$

Two pairs of symmetrical isoclines of Eq. (102) are illustrated in Fig. 2c for $\delta \Gamma_{e}= \pm 0.2 \%$ (the relative precision of Güttinger and Cannell's data) and $\delta \Gamma_{e}= \pm 0.02 \%$, respectively, thus evidencing the experimental challenge to validate the equation $\vartheta=Y_{c}\left(\frac{\mathcal{Z}_{\chi}^{+, 1}}{\mathbb{Z}_{\chi}^{+, 1}}\right)^{\frac{1}{\Delta}}$ at the $\%$-level when $\Delta \tau^{*} \lesssim 10^{-3}$. The needs for a "critical" increase of the experimental precision when $\Delta \tau^{*}$ decreases is now well quantified by Eqs. (98) and (102). These equations are essential when the objective is to test the asymptotic validity of the linearized Eq. (76) in presence of small but finite confluent corrections to scaling.

\section{References}

1. Anisimov, M.A., Sengers, J.V.: 11 Critical region. In: Sengers, J.V., Kayser, R.F., Peters, C.J., White, H.J., Jr. (eds.) Equations of State for Fluids and Fluid Mixtures, Part I, pp. 381-434. Elsevier, Amsterdam (2000)

2. Zinn-Justin, J.: Quantum Field Theory and Critical Phenomena, $4^{r d}$. University Press, Oxford (2002)

3. Bagnuls, C., Bervillier, C.: Nonasymptotic critical behaviour from field theory for Ising like systems in the homogeneous phase: theoretical framework. J. Phys. (Paris) Lett. 45, L95-L100 (1984)

4. Bagnuls, C., Bervillier, C.: Classical-to-critical crossovers from field theory. Phys. Rev. B 32, 7209 (1985)

5. Bagnuls, C., Bervillier, C.: Classical-to-critical crossovers from field theory. Phys. Rev. E 65, 066132-12p (2002). and references therein

6. Wilson, K.G., Kogut, J.: The renormalization group and the $\varepsilon$ expansion. Phys. Rep. 12(2), 75-200 (1974)

7. Privman, V., Hohenberg, P.C., Aharony, A.: Universal critical point amplitude relations. In: Domb, C., Lebowitz, J.B. (eds.) Phase Transitions and Critical Phenomena, pp. 1-134. Academic Press, New York (1991)

8. Guida, R., Zinn-Justin, J.: Critical exponents of the N-vector model. J. Phys. A 31, 8103-8122 (1998)

9. Garrabos, Y., Bervillier, C.: Mean crossover functions for uniaxial 3D Ising-like systems. Phys. Rev. E 74, 021113-16p (2006)

10. Wegner, F.J.: Corrections to scaling laws. Phys Rev. B 5, 4529-4536 (1972)

11. Garrabos, Y., Lecoutre-Chabot, C., Palencia, F., LeNeindre, B., Erkey, C.J.: Master crossover functions for one-component fluids. Phys. Rev. E 77(2), 021116 (2008)

12. Schloms, R., Dohm, V.: Minimal renormalization without $\varepsilon$-expansion: critical behavior in three dimensions. Nucl. Phys. B 328, 639-663 (1989)

13. Krause, H.J., Schloms, R., Dohm, V.: Minimal renormalization without $\varepsilon$-expansion: amplitude functions in three dimensions. Z. Phys. B 79, 287-293 (1990)

14. Chen, Z.Y., Albright, P.C., Sengers, J.V.: Crossover from singular critical to regular classical thermodynamic behavior of fluids. Phys. Rev. A 41, 3161-3177 (1990)

15. Anisimov, M.A., Kiselev, S.B., Sengers, J.V., Tang, S.: Crossover approach to global critical phenomena in fluids. Phys. A 188, 487-525 (1992)

16. Bagnuls, C., Bervillier, C., Garrabos, Y.: Experimental data analysis on xenon above the critical temperature from nonlinear renormalization group. J. Phys. (Paris) Lett. 45, L127-L132 (1984) 
17. Anisimov, M.A., Povodyrev, A.A., Kulikov, V.D., Sengers, J.V.: Nature of crossover between Ising-like and mean-field critical behavior in fluids and fluid mixtures. Phys. Rev. Lett. 75, 3146-3149 (1995)

18. Hahn, I., Zhong, F., Barmatz, M., Haussmann, R., Rudnick, J.: Crossover behavior in the isothermal susceptibility near the 3 He critical point. Phys. Rev. E 63, 055104(R)-4p (2001)

19. Zhong, F., Barmatz, M., Hahn, I.: Application of minimal substraction renormalization to crossover behavior near the 3He liquid-vapor critical point. Phys. Rev. E 67, 021106-20p (2003)

20. Güttinger, H., Cannell, D.S.: Corrections to scaling in the susceptibility of xenon. Phys. Rev. A 24, 3188-3201 (1981)

21. Garrabos, Y., Lecoutre, C., Marre, S., Guillaument, R., Beysens, D., Hahn, I.: Crossover equation of state models applied to the critical behavior of xenon. J. Stat. Phys. 158, 1379-1412 (2015)

22. Behnejad, H., Sengers, J.V., Anisimov, M.A.,In: Goodwin A.R.H., Sengers J.V., Peters C.J. (eds.) Applied Thermodynamics of Fluids, pp. 321-367. IUPAC, RSC Publishing, Cambridge (2010)

23. Hasenbusch, M.: A finite size scaling study of lattice models in the three-dimensional Ising Universality class. Phys. Rev. B 82, 174433 (2010)

24. Hasenbusch, M.: Universal amplitude ratios in the 3D Ising Universality class. Phys. Rev. B 82, 174434 (2010)

25. Pelissetto, A., Vicari, E.: Critical phenomena and renormalization group theory. Phys. Rep. 368, 549-727 (2002)

26. Kim, Y.C., Fisher, M.E., Orkoulas, G.: Asymmetric fluid criticality. I. Scaling with pressure mixing. Phys. Rev. E 67, 061506 (2003)

27. Barmatz, M., Zhong, F., Shih, A.: Reanalysis of Microgravity Heat Capacity Measurements near the $\mathrm{SF}_{6}$ Liquid-Gas Critical Point. Int. J. Thermophys. 25, 1667-1673 (2004)

28. Garrabos Y.: Contribution à l'étude des propriétés d'état des fluides purs dans leur région critique, Thèse de Doctorat d'état, Université Paris 6 (1982)

29. Garrabos Y.: Facteurs d'échelle phénoménologiques pour la transition critique liquide-gaz des fluides purs, J. Phys. (Paris) 46, 281 (1985) [for an english version see also: Phenomenological Scale Factors for the Liquid-Vapor Critical Transition of Pure Fluids, cond-mat/0512408]

30. Garrabos, Y.: Scaling behaviour of the fluid subclass near the liquid-gas critical point. J. Phys. (Paris) 47, 197-206 (1986)

31. Kim, Y.C., Anisimov, M.A., Sengers, J.V., Luijten, E.: Crossover critical behavior in the three-dimensional Ising model. J. Stat. Phys. 110, 591 (2003)

32. Garrabos, Y., Le Neindre, B., Wunenburger, R., Lecoutre-Chabot, C., Beysens, D.: Universal scaling form of the equation of state of a critical pure fluid. Int. J. Thermophys. 23, 997-1011 (2002)

33. Kouvel, J.S., Fisher, M.E.: Detailed magnetic behavior of nickel near its curie point. Phys. Rev. 136, A1626-A1632 (1964)

34. Närger, U., Balzarini, D.A.: Coexistence-curve diameter and critical density of xenon. Phys. Rev. B 42, 6651-6657 (1990)

35. Cornfeld, A.B., Carr, H.Y.: Experimental evidence concerning the law of rectilinear diameter. Phys. Rev. Lett. 29, 28-32 (1972). Erratum, 29, E320 (1972)

36. Balzarini, D., Mouritsen, O.G.: Universal ratio of correction-to-scaling amplitudes for Xe. Phys. Rev. A 28, 3515-3519 (1983)

37. Sengers, J.M.H.L., Greer, W.L., Sengers, J.V.: Scaled equation of state for gases in the critical region. J. Phys. Chem. Ref. Data 5, 1-51 (1976)

38. Fisher, M.E., Orkoulas, G.: The Yang-Yang anomaly in fluid criticality: experiment and scaling theory. Phys. Rev. Lett. 85, 696-699 (2000)

39. Garrabos, Y., Lecoutre-Chabot, C., Palencia, F., Erkey, C.J., LeNeindre, B.: Master singular behavior from correlation length measurements for seven one-component fluids near their gas-liquid critical point. Phys. Rev. E 73, 026125-9p (2006)

40. Le Guillou, J.C., Zinn-Justin, J.: Critical exponents for the N-vector model in three dimensions from field theory. Phys. Rev. Lett. 39, 95-98 (1977)

41. Sengers, J.L., Sengers, J.V.: Universality of critical behavior in gases. Phys. Rev. A 12, 2622-2627 (1975)

42. Levelt Sengers, J.M.H., Sengers, J.V.: Critical phenomena in classical fluids chap. 4. In: Croxton, C.A. (ed.) Progress in Liquid Physics, pp. 103-174. Wiley, New York (1978)

43. Green, M.S., Vicentini-Missoni, M., Levelt-Sengers, J.M.H.: Scaling-law equation of state for gases in the critical region. Phys. Rev. Lett. 18, 1113-1117 (1967)

44. Lecoutre, C., Guillaument, R., Marre, S., Garrabos, Y., Beysens, D., Hahn, I.: Weightless experiments to probe universality of fluid critical behavior. Phys. Rev. E 91, 060101(R)-5p (2015)

45. Garrabos, Y.: Universality and quantum effects in one-component critical fluid. Phys. Rev. E 73, 056110$13 p$ (2006) 
46. Garrabos Y.: Is the entropy at the liquid-gas critical point of pure fluids proportional to a master dimensionless constant?, hal-00016454 (2006)

47. Gillis, K.A., Shinder, I.I., Moldover, M.R.: Thermoacoustic boundary layers near the liquid-vapor critical point. Phys. Rev. E 70, 021201-20p (2004)

48. Gillis, K.A., Shinder, I.I., Moldover, M.R.: Bulk viscosity of stirred xenon near the critical point. Phys. Rev. E 72, 051201-20p (2005)

49. Berg, R.F., Moldover, M.R., Zimmerli, G.A.: Frequency-dependent viscosity of xenon near the critical point. Phys. Rev. E 60, 4079-4098 (1999)

50. Habgood, H.W., Schneider, W.G.: PVT measurements in the critical region of xenon. Can. J. Chem. 32, 98-112 (1954)

51. Habgood, H.W., Schneider, W.G.: Thermodynamic properties of xenon in the critical region. Can. J. Chem. 32, 164-173 (1954)

52. Weinberger, M.A., Schneider, W.G.: On the liquid-vapor coexistence curve of xenon in the region of the critical temperature. Can. J. Chem. 30, 422-422- (1952)

53. Weinberger, M.A., Schneider, W.G.: Density distributions in a vertical tube containing xenon near the critical temperature as measured by a radioactive tracer technique. Can. J. Chem. 30, 847-859 (1952)

54. Cannell, D.S., Benedek, G.B.: Brillouin Spectrum of Xenon Near Its Critical Point. Phys. Rev. Lett. 25, 1157-1161 (1970)

55. Smith, I.W., Giglio, M., Benedek, G.B.: Correlation range and compressibility of xenon near the critical point. Phys. Rev. Lett. 27, 1556-1559 (1971)

56. Baidakov, V.G., Rubshtein, A.M., Pomortsev, V.P., Sulla, I.J.: The equation of state of metastable liquid xenon near the critical point. Phys. Lett. A 131, 119-121 (1988)

57. Baidakov, V.G., Rubshtein, A.M., Pomortsev, V.P.: p, $\rho$, T-properties of stable and metastable xenon near the liquid-vapor critical point. Fluid Mech. Res. 21(3), 89-101 (1992)

58. Michels, A., Wassenaar, T., Louwerse, P.: Isotherms of xenon at temperatures between $0^{\circ} \mathrm{C}$ and $150^{\circ} \mathrm{C}$ and at densities up to 515 Amagats (pressures up to 2800 atmospheres). Physica 20, 99-106 (1954)

59. Wilcox, L.R., Balzarini, D.: Interferometric determination of near-critical isotherms of xenon in the earth's field. J. Chem. Phys. 48, 753-763 (1968)

60. Vicentini-Missoni, M., Levelt Sengers, J.M.H., Green, M.S.: Scaling analysis of thermodynamic properties in the critical region of fluids. J. Res. Natl. Bur. Stand. (USA) 73A, 563-583 (1969)

61. Ho, J.T., Lister, J.D.: Faraday rotation near the ferromagnetic critical temperature of $\mathrm{CrBr}_{3}$. Phys. Rev. B 2, 4523-4532 (1970)

62. Estler, W.T., Hocken, R., Charlton, T., Wilcox, L.R.: Coexistence curve, compressibility, and the equation of state of xenon near the critical point. Phys. Rev. A 12, 2118-2136 (1975)

63. Hohenberg, P.C., Barmatz, M.: Gravity effects near the gas-liquid critical point. Phys. Rev. A 6, 289-313 (1972)

64. Swinney, H.L., Henry, D.L.: Dynamics of fluids near the critical point: decay rate of order-parameter fluctuations. Phys. Rev. A 8, 2586-2617 (1973)

65. Berg, R.F., Moldover, M.R.: Critical exponent for the viscosity of carbon dioxide and xenon. J. Chem. Phys. 93, 1926-19 (1990)

66. Berg, R.F., Lyell, M.J., McFadden, G.B., Rehm, R.G.: Internal waves in xenon near the critical point. Phys. Fluids 8, 1464-1475 (1996)

67. Fisher, M.E., Zinn, S.-Y., Hupton, P.J.: Trigonometric models for scaling behavior near criticality. Phys. Rev. B 59, 14533-14545 (1999) 